\title{
Spin Many-Body Phases in Standard- and Topological-Waveguide QED Simulators
}

\author{
M. Bello®, ${ }^{1, *}$ G. Platero $\odot,{ }^{2}$ and A. González-Tudela $\odot^{3, \dagger}$ \\ ${ }^{1}$ Max-Planck-Institut für Quantenoptik, Hans-Kopfermann-Strasse 1, Garching 85748, Germany \\ ${ }^{2}$ Instituto de Ciencia de Materiales de Madrid, CSIC, Madrid 28049, Spain \\ ${ }^{3}$ Instituto de Física Fundamental, IFF-CSIC, Calle Serrano 113b, Madrid 28006, Spain
}

(Received 30 June 2021; revised 24 January 2022; accepted 31 January 2022; published 7 March 2022)

\begin{abstract}
Waveguide QED has emerged as a powerful analog quantum simulator due to the possibility of mediating versatile spin-spin interactions with tunable sign, range, and even dimerization. Yet, despite their potential, the many-body phases emerging from these systems have only been scarcely explored. Here, we characterize the many-body phases of a large class of spin models that can be obtained in such waveguideQED simulators and uncover, importantly, the existence of symmetry-protected topological phases with large-period magnetic orderings with no analog in other state-of-art simulators. We explain that these phases emerge from the unique combination of long-range and dimerized interactions appearing in these platforms and propose several experimental observables to characterize them. Finally, we also develop an adiabatic protocol to prepare such states and analyze its performance with the main decoherence source of these systems.
\end{abstract}

DOI: 10.1103/PRXQuantum.3.010336

\section{INTRODUCTION}

The importance of quantum spin problems in several areas of physics and computation is hard to overestimate [1]. For example, interacting spin systems are believed to be instrumental for understanding the behavior of high$T_{c}$ superconductors [2] or exciton transport [3], they are paradigmatic models of quantum phase transitions [4], and, beyond physics, their ground states can codify the solution of many NP-hard optimization problems [5]. Irrespective of the context in which they appear, understanding their many-body behavior represents an outstanding computational challenge due to the exponential growth of their Hilbert space with the system size. This difficulty becomes critical for frustrated spin systems [6], where the classical algorithms that avoid the exponential growth of resources required, such as Monte Carlo, do not perform well. An alternative way to study these problems consists in building analog quantum simulators-well-controlled systems that replicate the interactions of the models to

\footnotetext{
*miguel.bello@mpq.mpg.de
}

†a.gonzalez.tudela@csic.es

Published by the American Physical Society under the terms of the Creative Commons Attribution 4.0 International license. Further distribution of this work must maintain attribution to the author(s) and the published article's title, journal citation, and DOI. be understood [7]. In this way, by preparing, manipulating, and measuring the simulator, one can get information about the spin model that would be difficult to obtain otherwise.

Among the different platforms available, atomic systems in their different incarnations are nowadays the leading technology for analog simulation of quantum magnetism. For example, the internal levels of trapped-ion chains can behave as effective spin systems that interact through their collective motion [8], with interactions decaying generally as $1 / r^{3}$ with the distance $r$ between ions [9]. These simulators have been experimentally realized by many groups already [10-15], who have used them, for example, to probe the phase diagram of interacting spin chains [13]. Another implementation that can be used to simulate quantum spin models consists of neutral atoms trapped in optical lattices. To do so, one can define a pseudospin degree of freedom either by using the occupation difference between neighboring lattice sites [16-20] or the hyperfine levels of the atoms [21-28], leading to ZZ or Heisenbergtype interactions, respectively. Unfortunately, irrespective of the method chosen, the interaction range is restricted to nearest neighbors, limiting the frustration that can be obtained in these systems. To extend this range, one can excite the atom to a Rydberg state, yielding $1 / r^{6}$ (ZZ) or $1 / r^{3}(\mathrm{XX})$ interactions depending on the parity of the Rydberg state [29-34]. This increase in the interaction range comes at the price of introducing additional noise into the system due to the Rydberg-state lifetime, although it 
has still enabled the observation of novel frustrated spin many-body phases experimentally [32] coming from the longer-range nature of the interactions.

Despite the unquestionable success of all these platforms, the models they can simulate are limited by the interactions that appear naturally in these systems, which generally have a fixed range, sign, or type. WaveguideQED setups, where quantum emitters couple to onedimensional (1D) propagating fields [35-53], have been suggested as a powerful alternative paradigm to study quantum magnetism, circumventing these tunability limitations [54-60]. The key idea is that when the transition frequencies of the emitters are tuned to a photonic band gap, the photons localize around them, forming bound states [61-63] that can mediate coherent spin interactions between the emitters. The sign, range, and even dimerization of these bound-state-mediated interactions can be largely tuned by either properly engineering the waveguide or through a Raman-assisted process [54-59]. Besides, by combining several Raman-assisted processes, one can further tune the nature of the interactions from the naturally appearing exchange interactions to the Ising type [54] and combinations of them [55,56]. These exciting perspectives have triggered many experimental advances in the field and nowadays we count with many platforms where natural $[36,37,40,42-44]$ and artificial atoms [45-47], superconducting qubits [49-52], or even simulated emitters [53] couple to waveguides with photonic band gaps. Remarkably, despite their great potential for quantum simulation, the expected many-body phases that emerge in these systems have only been scarcely investigated. One of the first works on this issue is Ref. [64], which explores the interplay between the motion and the internal (spin) degrees of freedom of an ensemble of quantum emitters coupled to an undimerized photonic waveguide. Another interesting possibility is the simulation of disordered models, where the position of the emitters is random, as studied, for example, in Ref. [65].

In this paper, we cover this gap, providing the first complete analysis of the many-body spin phases that appear in a large variety of waveguide-QED setups. Based on previous works [54-59], we consider a generalized XXZ spin model with interactions that originate in a photonic Su-Schrieffer-Heeger (SSH) waveguide. This kind of waveguide can produce long-range dimerized (sometimes referred to as chiral) interactions with staggered sign and it describes both the trivial and topologicalwaveguide cases. Using density-matrix renormalizationgroup techniques $[66,67]$ and exact diagonalization, we find and characterize frustrated many-body phases with long-range magnetic order emerging from the competition of all these features and propose observables to characterize them experimentally. Importantly, we show that some of these phases are symmetry-protected topological phases displaying quantized many-body Berry phases [68-70]. Our results thus demonstrate the potential of waveguide-QED platforms as an analog quantum simulator of frustrated spin models, including those considering the interplay between topology and longrange interactions, which have aroused a lot of interest recently [71-74].

The paper is structured as follows. In Sec. II, we describe the model that we consider, explaining the shape of the waveguide-mediated interactions in different bandgap configurations. In Sec. III, we study the many-body phases that emerge in conventional (undimerized) waveguides, where both long-range and/or staggered-sign interactions appear, depending on the type of band gap chosen. Then, in Sec. IV, we consider the case of topological waveguides $[52,58,59]$, where, in addition, the dimerization of the interactions can be tuned by modifying the properties of the waveguide. In Sec. V C, we design and benchmark an adiabatic protocol to prepare the most relevant phases and study its resilience to one of the most relevant decoherence sources, that is, emitter excitation loss. Finally, we summarize our findings in Sec. VI and conclude in Sec. VII.

\section{GENERALIZED SPIN MODELS IN WAVEGUIDE QED}

The system we study consists of $N$ quantum emitters that interact locally with the photonic analog of the SSH model $[58,59]$. This waveguide can be described as a set of tunnel-coupled cavities with alternating hopping amplitudes $J(1 \pm \delta)$, all with the same resonant frequency $\omega_{a}$ [see Fig. 1(a)]. This model covers both the cases of conventional waveguides, if $\delta=0$, where a single energy band with a simple dispersion appears, $\omega(k)=$ $\omega_{a}-2 J \cos (k)$, and the case of dimerized waveguides, if $\delta \neq 0$, where the model displays two energy bands [see Fig 1(b)] and where the waveguide may have a nontrivial topology if $\delta<0$, according to our choice of the unit cell.

The emitters are coupled to the waveguide through an electric-dipole transition appearing between a ground and an optically excited state, which has a relative detuning $\Delta$ with respect to the mean frequency $\omega_{a}$ of the photons in the photonic waveguide. In the simplest scenario, these two levels define a qubit or spin, the dipole operator $\propto S_{n}^{x}$ of which couples with the electric field inside the cavity, $\propto\left(a+a^{\dagger}\right) ; a\left(a^{\dagger}\right)$ annihilates (creates) a photon in the cavity. Within the usual rotating-wave approximation, the interaction Hamiltonian describing this coupling is approximately $\sum_{n}\left(a^{\dagger} S_{n}^{-}+\right.$H.c. $)$[75]. Assuming that the transition frequencies of the emitters lie in the band gap and that the bath time scales are much faster than those for the emitters (Born-Markov conditions) [75], one can adiabatically eliminate the waveguide photons and 
(a)
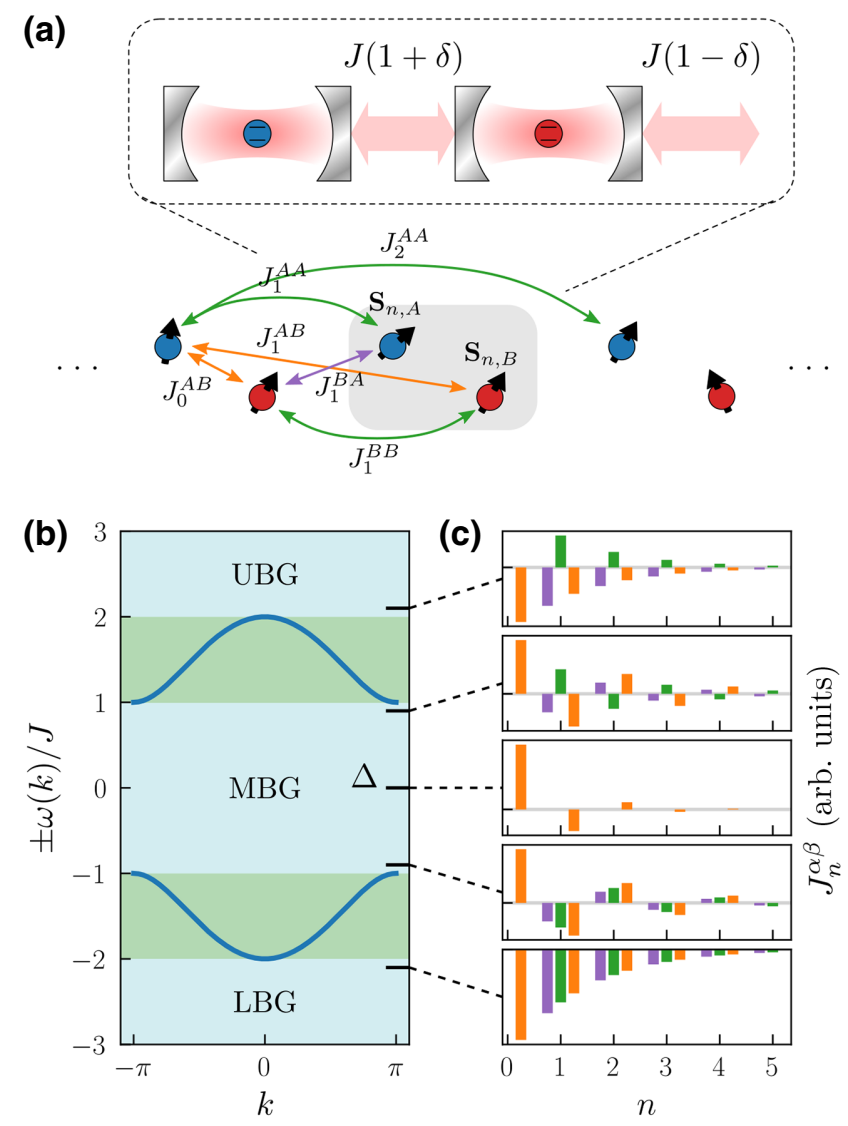

FIG. 1. Waveguide-mediated interactions. (a) A schematic of the system under consideration. The blue (red) dots represent spins in the $A(B)$ sublattice and the colored arrows represent couplings between different spins. (b) The energy bands of the photonic SSH model for $\delta=0.5$. The shaded blue areas mark the upper, middle, and lower band gaps, as opposed to the band ranges (green shaded areas). (c) The interaction constants as a function of the distance for different values of the emitter transition frequency $\Delta$, as indicated by horizontal segments on the right-hand side of plot (b). Different spin interactions are colored as in the system diagram (a). Positive (negative) coupling constants are plotted above (below) the gray horizontal line.

obtain a coherent spin exchange interaction approximately of the form $\left(S_{n}^{+} S_{m}^{-}+\right.$H.c. $)[54,55]$ for all emitter pairs. However, in atomic systems one can also define these spins with two hyperfine levels of the ground-state manifold and use Raman-assisted processes to couple them to the waveguide. In this way, one can obtain more general light-matter interaction Hamiltonians, e.g., approximately $\left(a^{\dagger} S_{n}^{x}+\right.$ H.c. $),\left(a^{\dagger} S_{n}^{z}+\right.$ H.c. $)$, etc., depending on the Raman configuration chosen [54-56] (Appendix A), which will result in different spin-interaction processes $\left(S_{n}^{x} S_{m}^{x}\right.$, $\left.S_{n}^{z} S_{m}^{z}, \ldots\right)$. Thus, for the sake of generality and to compare with previous works about other systems [76], we study the emergent many-body phases of the following generalized
XXZ model:

$$
\begin{aligned}
H= & -\sum_{j} \sum_{n \geq 0} \sum_{\alpha, \beta} J_{n}^{\alpha \beta}\left[\frac{\sin \theta}{2}\left(S_{j, \alpha}^{+} S_{j+n, \beta}^{-}+\text {H.c. }\right)\right. \\
& \left.+\cos \theta S_{j, \alpha}^{z} S_{j+n, \beta}^{z}\right]-\mu \sum_{\alpha} \sum_{j} S_{j, \alpha}^{z},
\end{aligned}
$$

written here in terms of spin-1/2 operators $S_{j, \alpha}^{v}$ and $v \in$ $\{x, y, z\}$ and ladder operators $S_{j, \alpha}^{ \pm}=S_{j, \alpha}^{x} \pm i S_{j, \alpha}^{y}$. The Latin subscripts label different unit cells, while Greek subscripts label different sublattices $\alpha, \beta \in\{A, B\}$ [for a schematic, see Fig. 1(a)]. For convenience, we sometimes use a single subscript to label the spins in the chain; the correspondence between the two labelings is given by $S_{2 j}^{\nu} \equiv S_{j, A}^{\nu}$ and $S_{2 j+1}^{\nu} \equiv S_{j, B}^{v}$. This generalized model includes:

(a) A tunable weight between the spin exchange and ZZ interactions, parametrized by an angle $\theta$, which can be physically obtained by tuning the weight of different Raman transitions [54-56]. For example, $\theta=$ $\pi / 2$ corresponds to standard exchange interactions that appear naturally in photon-mediated interactions, while $\theta=\pi / 4$ corresponds to the isotropic Heisenberg model.

(b) A parameter $\mu$ that plays the role of an external magnetic field or chemical potential that fixes the number of excitations in the ground state. Physically, this would correspond either to the energy of the optical transition, if one were to define the spin with the ground and excited states of the emitter; or to a tunable energy (through the frequencies of the Raman lasers) if one were to define the spin using the hyperfine levels of the emitter.

(c) Waveguide-mediated interactions with coupling constants $J_{n}^{\alpha \beta}$, which determine how spins $n$ unit cells apart interact. For simplicity, we assume that both the exchange and the $\mathrm{ZZ}$ terms have the same functional form. Importantly, the functional form of $J_{n}^{\alpha \beta}$ is dictated by the shape of the photonic component of the bound states, depending on the particular band gap with which the emitters are in resonance. For a nonzero dimerization $(\delta \neq 0)$, the energy spectrum of the SSH photonic bath displays three band gaps in the single-excitation sector spanning the ranges $(-\infty,-2 J),(-2 J|\delta|, 2 J|\delta|)$, and $(2 J, \infty)$, which we label as the lower (LBG), middle (MBG), and upper (UBG) band gap, respectively, as depicted in Fig. 1(b). Each one gives rise to qualitatively different coupling constants $J_{n}^{\alpha \beta}$, as shown in Fig. 1(c). There, we observe how it is possible to go from long-range antiferromagnetic interactions in the LBG to fully dimerized couplings in the MBG and staggered-sign long-range hoppings 
in the UBG, which demonstrates the potential of this platform to simulate a wide variety of spin models.

The exact form of the coupling constants, $J_{n}^{\alpha \beta}$ has been calculated in Ref. [58]. Interestingly, they can be parametrized by just two parameters (see Appendix A): the interaction length $\xi \in[0, \infty)$ and the effective dimerization constant:

$$
\tilde{\delta} \equiv \frac{\left|J_{n}^{A B}\right|-\left|J_{n+1}^{B A}\right|}{\left|J_{n}^{A B}\right|+\left|J_{n+1}^{B A}\right|} \in[-1,1] .
$$

For convenience, we also define the ratio between coupling constants connecting spins in the same or a different sublattice:

$$
\eta \equiv \frac{2\left|J_{n+1}^{A A}\right|}{\left|J_{n}^{A B}\right|+\left|J_{n+1}^{B A}\right|}=\left[e^{-1 / \xi}\left(1-\tilde{\delta}^{2}\right)\right]^{1 / 2} \in[0,1] .
$$

In terms of these parameters, the coupling constants read as follows:

$$
\begin{aligned}
J_{n}^{A B} & =-\tilde{J}(1+\tilde{\delta}) e^{-n / \xi}, \\
J_{n}^{B A} & =-\tilde{J}(1-\tilde{\delta}) e^{-(n-1) / \xi}, \\
J_{n}^{A A / B B} & =\tilde{J} \operatorname{sign}(\Delta) \eta e^{-(n-1) / \xi},
\end{aligned}
$$

for $|\Delta|>2 J$ (outer band gaps, UBG and LBG), while

$$
\begin{aligned}
J_{n}^{A B} & =\tilde{J} \operatorname{sign}(\tilde{\delta})(1+\tilde{\delta})(-1)^{n} e^{-n / \xi}, \\
J_{n}^{B A} & =-\tilde{J} \operatorname{sign}(\tilde{\delta})(1-\tilde{\delta})(-1)^{n-1} e^{-(n-1) / \xi}, \\
J_{n}^{A A / B B} & =\tilde{J} \operatorname{sign}(\Delta) \eta(-1)^{n-1} e^{-(n-1) / \xi},
\end{aligned}
$$

for $|\Delta|<2 J|\delta|$ (MBG); $n \geq 1$, except for $J_{0}^{A B}$, which is the only nonzero coupling constant between spins that belong to the same unit cell. $\tilde{J}$ is the overall coupling strength, which is specific to each implementation. In the case of emitters with a single dipolar transition, it behaves as $\tilde{J} \sim g^{2} / \sqrt{\Delta_{\text {edge }}}$, where $g$ denotes the lightmatter coupling strength and $\Delta_{\text {edge }}=\left|\Delta-\omega_{\text {edge }}\right|$ is the distance (in frequency) from the transition frequency of the emitters to the closest band edge. In some experimental platforms, such as superconducting circuits, these quantities can be tuned directly, while in others, they depend on other experimentally accessible parameters. This is the case, for example, for cold atoms in state-dependent optical lattices [77,78] or atomic arrays near photonic crystals [54,79], both of which use Raman lasers (external drivings) to modify the optical properties of the emitters. In those cases, $g$ and $\Delta_{\text {edge }}$ will depend on the amplitudes and frequencies of the Raman lasers.

The values of $\xi$ and $\tilde{\delta}$ can be tuned to a great extent by tuning different system parameters. The interaction length diverges as $\xi \sim-1 / \sqrt{\Delta_{\text {edge }}}$ as the emitter detuning gets closer to any band edge, while $\operatorname{sign}(\tilde{\delta})=\operatorname{sign}(\delta)$, implying that both the effective spin model and the underlying bath have a similar dimerization pattern (for a detailed account of their dependence on the different system parameters, see Appendix A). However, each value of the pair $(\xi, \tilde{\delta})$ is possible only in either the outer band gaps or in the inner band gap but not both. In the outer band gaps $|\tilde{\delta}|>\left(1-e^{-1 / \xi}\right) /\left(1+e^{-1 / \xi}\right)$, whereas in the inner band gap the same inequality is fulfilled, replacing " $>$ " by " $<$." Consequently, in the outer band gaps, interactions connecting spins in the same sublattice are weaker than those connecting spins in different sublattices, $\eta<(1-|\tilde{\delta}|)$; in the MBG, by contrast, their strength lies in between, $(1-$ $|\tilde{\delta}|)<\eta<(1+|\tilde{\delta}|)$, so the system resembles a ladder more than a simple $1 \mathrm{D}$ chain.

In the following sections, we analyze the many-body phases emerging from this generalized spin Hamiltonian, starting in Sec. III with the case of a standard waveguide, where $\delta=0$ and only the LBG and UBG exist. Then, in Sec. IV, we move to the more complex scenario with $\delta \neq 0$. But before doing so, we point out to the reader two important symmetries of the model. The Hamiltonian is invariant under rotations about the $z$ axis of all the spins forming the chain; consequently, the total magnetization $m=\sum_{j, \alpha} S_{j, \alpha}^{z}$ is conserved and we can reduce the problem of finding the ground state of the system to the calculation of the ground state within each fixed-magnetization subspace. Furthermore, it suffices to do so for $\mu=0$, since the chemical potential term is proportional to the identity operator in each fixed-magnetization subspace (the eigenstates of $H$ are the same for all values of $\mu$ and the eigenenergies depend linearly on $\mu$ at an $m$-dependent rate). Another symmetry of the model is the invariance of the Hamiltonian under the simultaneous flip of all spins accompanied by the change $\mu \rightarrow-\mu$. Thus, we can limit the study to the phases that appear for $\mu>0$.

Lastly, we point out that in order to characterize the different many-body phases, we mostly use correlation functions of the form $\left\langle S_{j, \alpha}^{\nu} S_{j, \beta}^{v}\right\rangle$ or $\left\langle S_{j, \alpha}^{\nu} S_{j, \beta}^{\nu}\right\rangle-\left\langle S_{j, \alpha}^{\nu}\right\rangle\left\langle S_{j, \beta}^{v}\right\rangle$ (this last form is usually referred to as "quantum correlations"). Throughout the paper, we use the notation $\langle O\rangle$ to denote the expectation value of the operator $O$ in the ground state. Since the ground states of the models here studied have a definite number of excitations, the expectation values $\left\langle S_{j, \alpha}^{x}\right\rangle=\left\langle S_{j, \alpha}^{y}\right\rangle=0$, so both forms of the correlation functions coincide for $v=x, y$. For $v=z$, however, the expectation values $\left\langle S_{j, \alpha}^{z}\right\rangle$ might differ from zero.

\section{MANY-BODY PHASES IN STANDARD-WAVEGUIDE QED}

In this section, we analyze the ground states of the generalized XXZ Hamiltonian in the case where the waveguide mediating the interactions has no dimerization $(\delta=0$, 
also implying $\tilde{\delta}=0$ ). In that case, the MBG disappears and the waveguide-mediated interactions in the LBG and UBG simplify to

$$
\begin{aligned}
J_{n}^{A B} & =-\tilde{J} e^{-n / \xi}, \\
J_{n}^{B A} & =-\tilde{J} e^{-(n-1) / \xi}, \\
J_{n}^{A A / B B} & =\tilde{J} \operatorname{sign}(\Delta) e^{-1 /(2 \xi)} e^{-(n-1) / \xi} .
\end{aligned}
$$

Alternatively, if we use a single-index labeling of the spins along the chain, the coupling constant giving the strength of the interaction between the $i$ th and $j$ th spin is

$$
J_{i j}=-\tilde{J}[-\operatorname{sign}(\Delta)]^{|i-j|-1} e^{\frac{1-|i-j|}{2 \xi}}, i \neq j .
$$

In this regime, the main differences of the waveguide-QED simulator as compared with other analog spin simulators are the possibility of obtaining longer-range interactions, since $\xi$ can be tuned to be very large [54], and the appearance of staggered-sign interactions in the UBG. In the following, we first analyze what happens in the limiting cases of nearest-neighbor (NN) (in Sec. III A) and infinite-range interactions (in Sec. III B), which are exactly solvable and which help us to better understand the phases that appear for intermediate-range interactions, studied in Sec. III C. In addition, the case of infinite-range interactions is interesting because it connects with the spin models that can be obtained in cavity-QED setups $[80,81]$.

\section{A. Nearest-neighbor models}

The limit of NN interactions is the same for both the models in the upper and lower band gaps: $J_{0}^{A B}=J_{1}^{B A}=$ $-\tilde{J}$, while the rest of the couplings are zero. It occurs when $\Delta$ is far detuned from the energy band of the bath. This limit corresponds to the well-known XXZ Heisenberg model, an integrable model that can be solved by Bethe ansatz techniques [82]. Its phase diagram is shown in Fig. 2(a). Three distinct phases can be distinguished:

(1) Ferromagnetic phase. The ground state is the state in which all spins are aligned in the $z>0$ direction for $\mu>0$, which we can pictorially represent as $\uparrow \uparrow \uparrow \uparrow \ldots$. It is a product state and, therefore, it features no quantum correlations whatsoever between any two spins. The elemental excitations correspond to a single spin flip, $\uparrow \uparrow \downarrow \uparrow \uparrow \ldots .$. If the $x x$ - and $y y$ interaction terms are nonzero, these excitations can hop between neighboring spins, so the eigenstates of the $m=N / 2-1$ subspace are spin waves that carry spin 1, also known as magnons. Assuming that $\mu>0$, the single-magnon spectrum is given by

$$
\frac{\epsilon(k)}{\tilde{J}}=\sin \theta \cos k+\cos \theta\left(\frac{N}{4}-1\right)-\frac{\mu}{\tilde{J}}\left(\frac{N}{2}-1\right),
$$

(a)
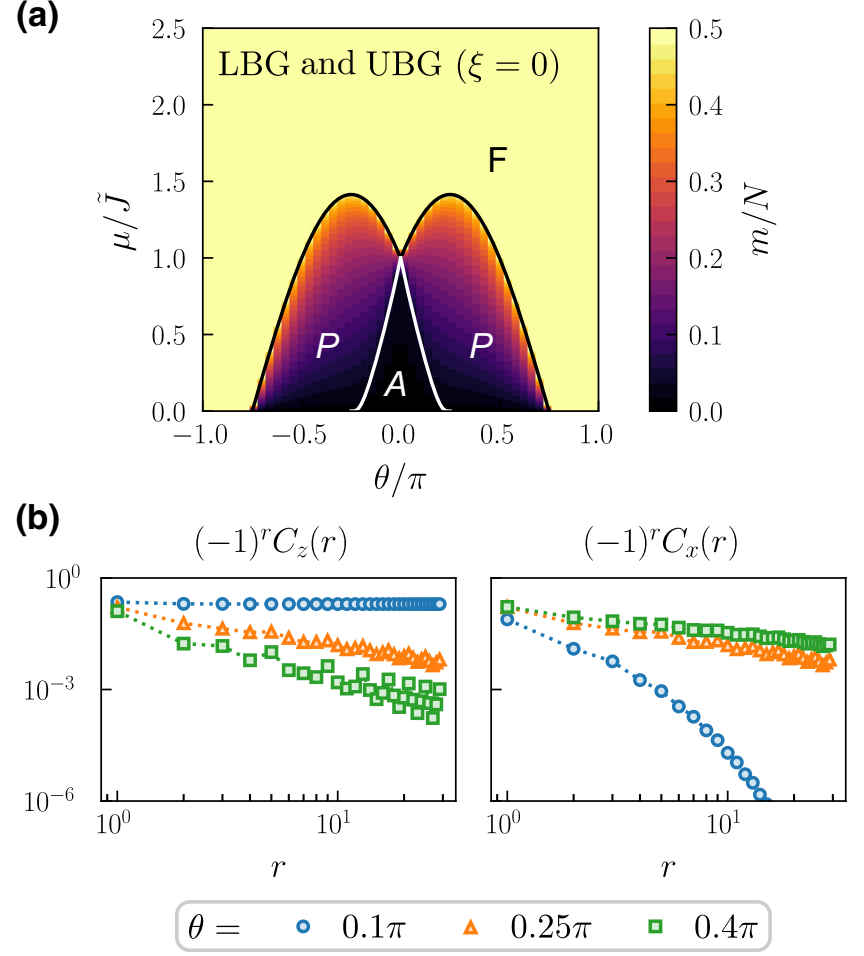

FIG. 2. The nearest-neighbor XXZ model $(\xi=0, \tilde{\delta}=0)$. (a) The ground-state magnetization, $m \equiv\left\langle\sum_{j, \alpha} S_{j, \alpha}^{z}\right\rangle$, as a function of the external magnetic field and the anisotropy angle for the nearest-neighbor XXZ spin model. The results are obtained using the density matrix renormalization group algorithm for a chain with $N=50$ spins and open boundary conditions (OBCs). On top of this, we plot the analytically obtained boundaries (black and white lines) of the antiferromagnetic (A), ferromagnetic (F), and planar (P) phases in the thermodynamic limit. (b) The quantum correlations $C_{v}(r)=\left\langle S_{N / 2}^{v} S_{N / 2+r}^{\nu}\right\rangle-\left\langle S_{N / 2}^{\nu}\right\rangle\left\langle S_{N / 2+r}^{v}\right\rangle$ in the ground state with zero magnetization $(m=0)$ and different values of the anisotropy angle. In the antiferromagnetic phase $(\theta=0.1 \pi)$, the correlations on the $z$ axis reach a constant value, while the correlations in the $x-y$ plane decay exponentially. At the isotropic point $(\theta=\pi / 4)$, the correlations in any spin direction are the same and they decay algebraically. In the planar phase $(\theta=0.4 \pi)$, correlations also decay algebraically but they are larger in the $x-y$ plane than on the $z$ axis.

where $k$ is the magnon quasimomentum. Computing the minimum of this dispersion relation, one can readily find the energy gap between the ground state and the first excited state as $\Delta E / \tilde{J}=$ $-|\sin \theta|-\cos \theta+\mu / \tilde{J}$. The critical values of the model parameters at which there is a phase transition from the ferromagnetic phase to other phases of the model are the points where $\Delta E=0$ [shown as a black curve in Fig. 2(a)].

(2) Néel phase (aka antiferromagnetic phase). At the point $\theta=0$, for positive $z z$ interactions, the classical ground state is the state in which spins are pointing in opposite directions in an alternating fashion, 
$\uparrow \downarrow \uparrow \downarrow \ldots$, also known as antiferromagnetic order. The lowest-energy excitations in this phase correspond to spinons, fermionic quasiparticles that carry spin $1 / 2$. They correspond to domain walls between the two possible antiferromagnetic orderings $\uparrow \downarrow \uparrow \downarrow \downarrow \uparrow \downarrow \uparrow \ldots$ The magnetization can only change in integer steps, so these excitations are created in pairs as the magnetization increases. In the vicinity of the Ising limit, for small, finite $x x$ and $y y$ interactions, this picture is still valid. The ground state features long-range antiferromagnetic correlations and the elemental excitations are spinons, which can now hop along the chain by two sites. Using perturbation theory [83], one can compute the energy of two spinons (states that belong to the subspace with $m=1)$ as $\epsilon\left(k_{1}\right)+\epsilon\left(k_{2}\right)$, where

$$
\frac{\epsilon(k)}{\tilde{J}}=-\frac{\cos \theta}{4}(N-2)+\sin \theta \cos 2 k-\frac{\mu}{2 \tilde{J}} .
$$

They form a continuum of scattering eigenstates. Therefore, close to the classical antiferromagnetic Ising model $(\theta \rightarrow 0)$, the energy gap is $\Delta E / \tilde{J} \approx$ $\cos \theta-2|\sin \theta|-\mu / \tilde{J}$, which gives a good approximation of the critical line shown in Fig. 2(a) in white. The exact energy gap in the thermodynamic limit can be obtained using the Bethe ansatz [84], which is the formula we use in Fig. 2(a).

(3) Planar phase (aka XY phase or Tomonaga-Luttinger phase). This phase is fundamentally different from the previous two, as the system is gapless, i.e., excitations over the ground state can be produced at arbitrary small energies. In these situations, the system is said to be in a critical phase. This can be better understood at the XX limit $(\theta \rightarrow \pi / 2)$, where the spin chain can be mapped to a system of free fermions hopping on a 1D lattice via the JordanWigner transformation [85] (see Appendix E). These fermions have the familiar dispersion relation $\epsilon(k)=2 \tilde{J} \cos (k)-\mu$ and the ground state can be constructed as the Slater determinant of all singleparticle states with energies $\epsilon(k)<0$. For $|\mu|<2 \tilde{J}$, the Fermi level lies somewhere in the middle of the energy band and excitations can be created adding fermions with energies above the Fermi level (possibly removing them from energy states below it). Being a critical phase, correlations decay as a power law (algebraically), whereas in gapped phases they decay exponentially with increasing distance [see Fig. 2(b)]. In contrast to the (anti)ferromagnetic phases, correlations in this phase occur predominantly in the $x-y$ plane. Away from the XX limit, the Jordan-Wigner transformation maps the spin chain to a model of interacting fermions in $1 \mathrm{D}$, which is the subject of the so-called Tomonaga-Luttinger theory [86].

Since the system is bipartite - interactions only involve spins that belong to different sublattices - the phase diagram is symmetric with respect to the change $\theta \rightarrow-\theta$. To understand why, note that a transformation with $U=$ $\prod_{j} i 2 S_{j, A}^{z}$ inverts the sign of the flip-flop terms of the Hamiltonian (i.e., interactions in the $x-y$ plane), while it preserves the total magnetization.

\section{B. Infinite-ranged interactions}

The addition of couplings beyond nearest neighbors breaks the sublattice symmetry. The system is no longer bipartite - spins couple to other spins in the same and the opposite sublattice - making the phase diagram asymmetric with respect to the change $\theta \rightarrow-\theta$. This asymmetry can be clearly appreciated in the limit of infinite-range interactions, shown in Figs. 3(a) and 3(b). These models occur in the upper and lower band gaps as the emitter transition frequency gets closer to the band edges ( $\Delta \rightarrow-2 \tilde{J}$ for the LBG and $\Delta \rightarrow 2 \tilde{J}$ for the UBG). We should remark, however, that the Markovian approximation becomes worse as the frequency of the emitters gets closer to the band edges, so this limit would be hard to achieve in actual experiments. In any case, understanding the physics of these models allows us to better comprehend what happens at intermediate-interaction ranges.

In the LBG, the coupling constants of the infiniterange model are $J_{n}^{\alpha \beta}=-\tilde{J}$ for all $n$, so the Hamiltonian can be written in terms of the collective spin operators $S^{\nu} \equiv \sum_{n, \alpha} S_{n, \alpha}^{\nu}, \nu \in\{+,-, z\}$, as

$$
H=\frac{\tilde{J}}{2}\left[\frac{\sin \theta}{2}\left(S^{+} S^{-}+S^{-} S^{+}\right)+\cos \theta\left(S^{z}\right)^{2}\right]-\mu S^{z},
$$

apart from an unimportant constant term. The eigenstates of this Hamiltonian are the Dicke states $|s, m, \lambda\rangle$, which are also eigenstates of the collective spin operators $\mathbf{S}^{2}$ and $S^{z}$ with eigenvalues $s(s+1)$ and $m$, respectively [87]. The energies of these states are given by

$$
\begin{aligned}
E(s, m) & \equiv\langle s, m, \lambda|H| s, m, \lambda\rangle \\
& =\frac{\tilde{J}}{2}\left\{\sin \theta\left[s(s+1)-m^{2}\right]+\cos \theta m^{2}\right\}-\mu m .
\end{aligned}
$$

The phase diagram shown in Fig. 3(a) can be obtained minimizing $E(s, m)$ :

(a) For $\theta<0$, the ground state has maximum total angular momentum $s=N / 2$. The ground state is, thus, nondegenerate and invariant under any 


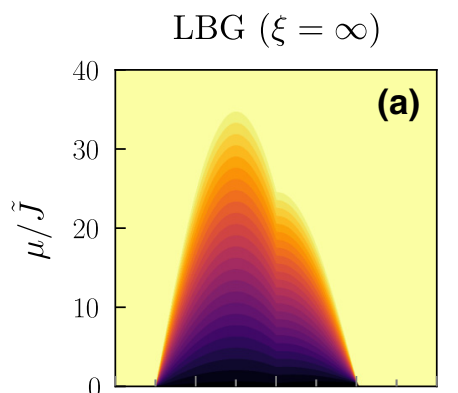

$\mathrm{UBG}(\xi=\infty)$
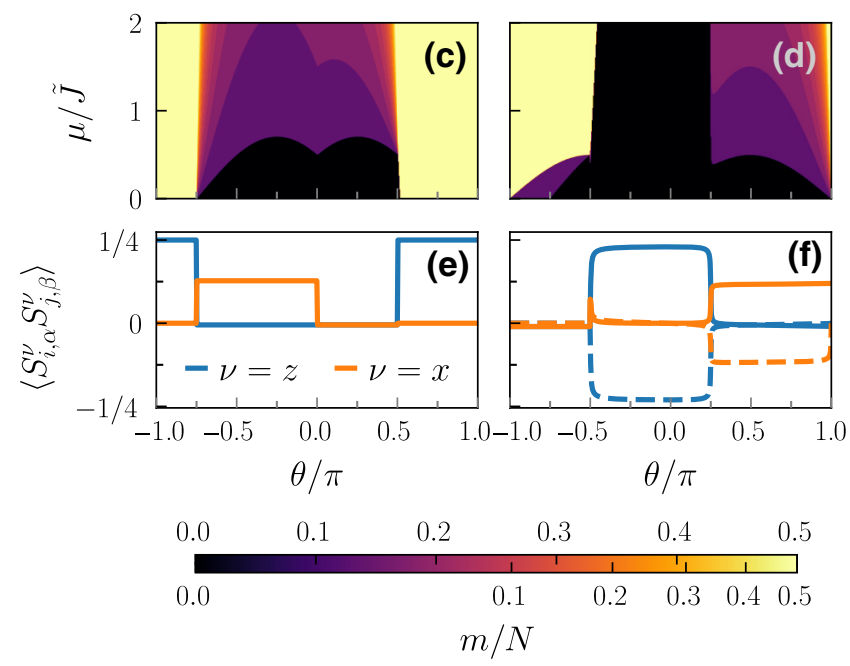

FIG. 3. Undimerized infinite-interaction-range models in the LBG and UBG. (a),(b) Ground-state-magnetization phase diagrams for a chain with $N=50$ spins. (c),(d) An enlargement of the phase diagrams for small values of $\mu$. In order to distinguish the different magnetization sectors, the color scale used (ticks under the color bar) is different from the one used in the upper panels (ticks above the color bar). (e),(f) Correlations in the ground state for $\mu \rightarrow 0^{+}$; the continuous (dashed) lines in panel (f) show the correlations between spins that belong to the same (opposite) sublattice.

permutation of the spins in the chain. Consequently, the correlations are the same regardless of the distance separating the spins. For a ground state with magnetization $m$, they are given by

$$
\begin{aligned}
& \left\langle S_{i, \alpha}^{z} S_{j, \beta}^{z}\right\rangle=\frac{4 m^{2}-N}{4 N(N-1)}, \\
& \left\langle S_{i, \alpha}^{x} S_{j, \beta}^{x}\right\rangle=\left\langle S_{i, \alpha}^{y} S_{j, \beta}^{y}\right\rangle=\frac{N^{2}-4 m^{2}}{8 N(N-1)},
\end{aligned}
$$

for all $(i, \alpha) \neq(j, \beta)$.

(b) For $\theta>0$, the ground state has minimum total angular momentum compatible with the groundstate magnetization, $s=m$. Thus, the ground state is degenerate for all values of $m$, except for $m=$ $N / 2$ (ferromagnetic phase). This degeneracy is associated with the permutation quantum number $\lambda$ [87]. Nevertheless, if we consider a fully mixed state within the ground-state manifold (a thermal state $\rho \propto e^{-\beta H}$ in the limit $\beta \rightarrow \infty$ ), the correlations become invariant under any permutation of the spins forming the chain; the $z z$ correlations in this case are the same as in Eq. (18), while the $x x$ and $y y$ correlations are given by

$$
\left\langle S_{i, \alpha}^{x} S_{j, \beta}^{x}\right\rangle=\left\langle S_{i, \alpha}^{y} S_{j, \beta}^{y}\right\rangle=\frac{2 m-N}{4 N(N-1)} .
$$

Note that in this model, all spins have the same polarization, $\left\langle S_{i, \alpha}^{z}\right\rangle=m / N$, regardless of the value of $\theta$.

As for the infinite-range model in the UBG, the coupling constants are $J_{n}^{A B / B A}=-J_{n}^{A A / B B}=-\tilde{J}$ for all $n$. The Hamiltonian can be written in this case as

$$
\begin{aligned}
H= & -\frac{\tilde{J}}{2} \sum_{\alpha=A, B}\left[\frac{\sin \theta}{2}\left(S_{\alpha}^{+} S_{\alpha}^{-}+S_{\alpha}^{-} S_{\alpha}^{+}\right)+\cos \theta\left(S_{\alpha}^{z}\right)^{2}\right] \\
& +\tilde{J}\left[\frac{\sin \theta}{2}\left(S_{A}^{+} S_{B}^{-}+S_{A}^{-} S_{B}^{+}\right)+\cos \theta S_{A}^{z} S_{B}^{z}\right] \\
& -\mu\left(S_{A}^{z}+S_{B}^{z}\right),
\end{aligned}
$$

with collective spin operators $S_{\alpha}^{\nu} \equiv \sum_{n} S_{n, \alpha}^{\nu}$ and $v \in$ $\{+,-, z\}$. This Hamiltonian preserves the total angular momentum in each sublattice $s_{A}$ and $s_{B}$ and the total magnetization $m$. Its matrix representation in the subspace with fixed $\left(s_{A}, s_{B}, m\right)$ can be cast into tridiagonal form (see Appendix $C$ ), so it can be easily diagonalized for a large number of spins. In Fig. 3(b), we show the phase diagram obtained for a chain with $N=50$ spins. As it happens in the infinite-interaction-range model in the LBG, for the infinite-interaction-range model in the UBG the degeneracy of the ground state also depends on the ration of $z z$ to flip-flop interactions:

(a) For $-\pi / 2<\theta<\pi$, the ground state is unique and is fully symmetric under permutations of spins within each sublattice. Thus, correlations do not depend on the distance separating the spins but are different for spins within the same or the opposite sublattice. In contrast to the infinite-interactionrange model in the LBG, they do not have a simple expression in terms of the magnetization $m$ and the total number of spins $N$ forming the chain but they can be easily computed numerically (for further details, see Appendix C). In some cases, the ground state can be computed analytically. For example, at the isotropic (Heisenberg) point $\theta=\pi / 4$, the ground state for $\mu=0$ is a singlet of total angular momentum $\mathbf{S}^{2}=\left(\mathbf{S}_{A}+\mathbf{S}_{B}\right)^{2}$ and the correlations are

$$
\left\langle S_{i, A}^{\nu} S_{j, B}^{\nu}\right\rangle=-\frac{N+4}{12 N},\left\langle S_{i, \alpha}^{v} S_{j, \alpha}^{\nu}\right\rangle=\frac{1}{12} .
$$


(b) For $-\pi<\theta<-\pi / 2$, the correlations are not uniquely determined, since the ground state is degenerate. But if we consider a thermal state in the zero-temperature limit, they become homogeneous and can be computed analytically (see Appendix C).

In these infinite-range models, both in the UBG and LBG, the energy needed to flip a spin becomes an extensive quantity, since each spin interacts equally strongly with all the other spins. Consequently, the stability region of each phase with a fixed magnetization shifts to higher values of the external magnetic field $\mu$ as we increase the number $N$ of spins forming the chain. Eventually, as we approach the thermodynamic limit $N \rightarrow \infty$, the groundstate phases for any finite $\mu$ correspond to those appearing in the limit $\mu \rightarrow 0^{+}$. To better see what phases appear in that limit, in Figs. 3(c) and 3(d) we show an enlargement of the phase diagrams for small values of $\mu$. The corresponding ground-state correlations in this limit are shown in Figs. 3(e) and 3(f). As we have mentioned already, they do not depend on the distance separating the spins in the chain.

In short, the dimensionality of the model becomes irrelevant in the infinite-interaction-range limit. Although this behavior is very different from that of correlations in the $\mathrm{NN}$ model, some phases are very similar, if not the same: the ferromagnetic phase, which in the thermodynamic limit only occurs in the LBG for $\pi / 2<\theta<5 \pi / 4$ [the yellow region in the phase diagram shown in Fig. 3(c)]; and the antiferromagnetic phase present in the UBG for $-\pi / 2<$ $\theta<\pi / 4$ [the middle black region in the phase diagram shown in Fig. 3(d)].

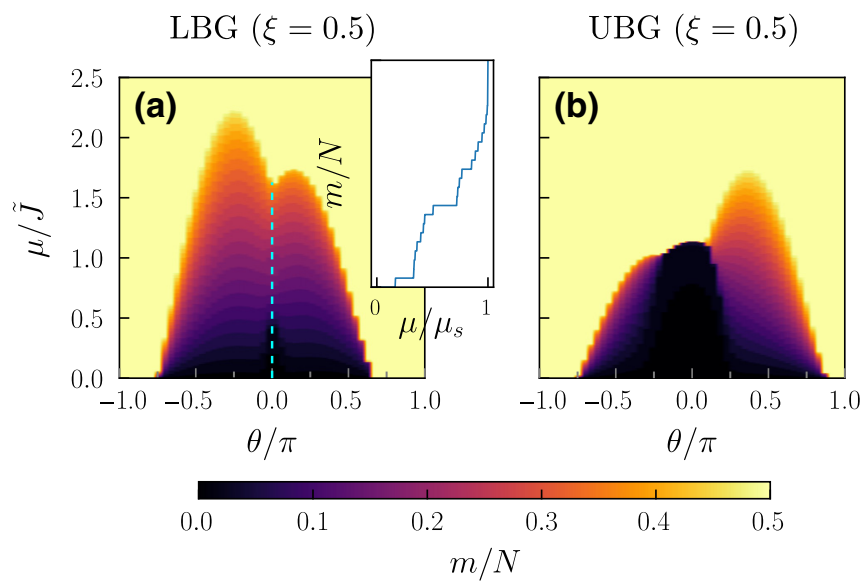

\section{Waveguide-mediated interactions: Intermediate ranges}

For intermediate-interaction lengths, we may expect the phase diagram to interpolate between that of the nearestneighbor XXZ Heisenberg model and the ones in the infinite-range interaction limit. This can be clearly appreciated in Figs. 4(a) and 4(b) [cf. Fig. 2 and Figs. 3(a), 3(b)]. In the light of these results, we can see that increasing the interaction length in the LBG shrinks the antiferromagnetic phase, confining it to values of $\theta$ closer to the classical limit $(\theta=0)$, while in the UBG it enlarges it, making this phase more stable against quantum fluctuations. This is also confirmed by the long-range $z z$ correlations [see Figs. 4(c) and 4(d) (blue dots)].

However, this is not the end of the story, as there are other phases that appear only when the interaction range is large but finite. For example, in the LBG, the phase diagram develops a series of magnetization plateaus [see the inset of Fig. 4(a)] around the classical regime $\theta=0$, which in the thermodynamic limit have a fractal structure known as a Devil's staircase $[76,88]$. These plateaus correspond to Ising-like phases with periodic $z z$ correlations with different periods (patterns).

Also, in the LBG, for positive exchange interactions $(0<\theta<\pi)$, there is a phase transition in the sector with zero total magnetization (small values of $\mu$ ) from a planar phase to a gapped spontaneously dimerized phase. This effect of frustration has been extensively studied in the past in simpler models, such as the antiferromagnetic zigzag Heisenberg chain [89-93]. A model where this phenomenon can be easily understood is the exactly solvable Majumdar-Ghosh model [1], the ground state of which is
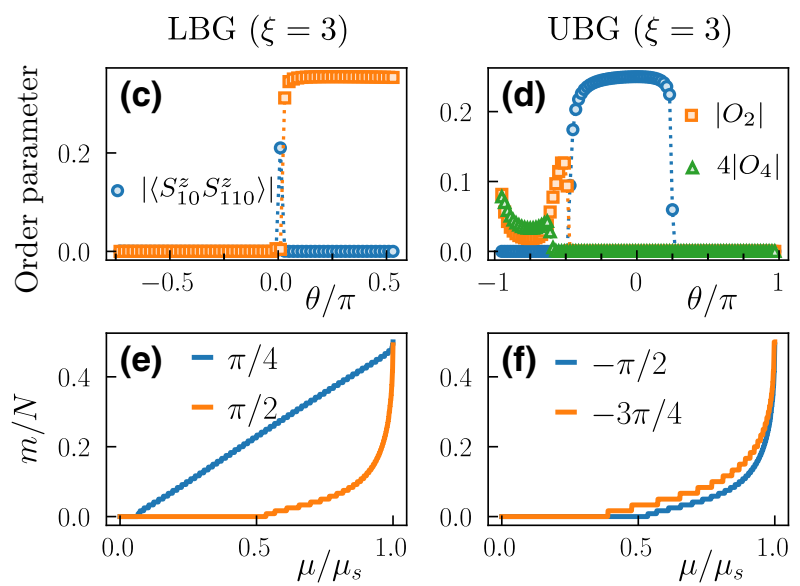

FIG. 4. Undimerized intermediate-interaction-range models in the LBG and UBG (a),(b) Ground-state-magnetization phase diagrams for a chain with $N=50$ spins. In (a), the inset shows the magnetization curve for a system close to the classical limit $(\theta \simeq 0$, dashed vertical line). (c),(d) Different order parameters as a function of the anisotropy angle in the ground state with zero magnetization, for a chain with $N=120$ spins (the legend is the same for both plots; in the LBG, the values of $\left|O_{4}\right|$ computed are negligible). (e),(f) Magnetization curves for a system with the same parameters as in panels (c) and (d), for particular values of $\theta$, as indicated in the legend. Here, $\mu_{s}$ denotes the saturation field, i.e., the value of $\mu$ at which the system becomes fully polarized. Note that this $\mu_{s}$ is a function of $\theta$. 
a product of singlets between adjacent spins. Phases of this kind are known as valence-bond solid (VBS) phases. Generally, these phases are gapped phases that do not feature any long-range order, i.e., the correlations $\left\langle S_{i}^{\nu} S_{j}^{\nu}\right\rangle$ decay exponentially with increasing distance $|i-j|$. However, fixing the distance, they become periodic as different spins along the chain are considered. In the spontaneously dimerized VBS phase appearing in the LBG, the correlations between neighboring spins (bond correlations) have period $p=2$ and $\left\langle B_{j}^{v}\right\rangle \equiv\left\langle S_{j}^{\nu} S_{j+1}^{v}\right\rangle \simeq \overline{B^{v}}+(-1)^{j} \delta B^{v}$ [94]. This dimer order in the bond correlations, as well as other orders with a larger spatial periodicity, can be detected experimentally by measuring spin correlations along the chain and computing the following quantity (the bond-order parameter or structure factor):

$$
O_{p}=\frac{1}{L} \sum_{n=n_{0}}^{n_{0}+L-1}\left\langle\mathbf{S}_{n} \mathbf{S}_{n+1}\right\rangle e^{-i \frac{2 \pi}{p} n} .
$$

In particular, the spontaneous dimerization can be quantified by $\left|\mathrm{O}_{2}\right|$. In Figs. 4(c) and 4(d), we plot this order parameter (orange squares) for the ground state with zero magnetization $(m=0)$. Its nonzero value for $0<\theta<\pi / 2$ in the LBG, and $-\pi<\theta<-\pi / 2$ in the UBG, proves the existence of dimer-VBS phases for sufficiently large but finite interaction length. For higher magnetization, $m \neq 0$, the value of $\left|O_{2}\right|$ is negligible.

Curiously, in the UBG we also find a VBS phase with a doubled period (tetramer order) for values of the anisotropy angle $\theta \approx-3 \pi / 4$ [see Fig. 4(d) (green triangles)]. Furthermore, in the magnetization curve shown in Fig. 4(f), we observe that the magnetization changes in steps of $\Delta m=2$ as $\mu$ increases. This is in contrast to the other cases studied, in which the magnetization increases in steps of $\Delta m=1$, cf. Fig. 4(e). Considering that in this case the couplings between spins in opposite sublattices are ferromagnetic, this suggests that excitations correspond in this tetramer phase to simultaneous flips of two spins, one in each sublattice. These gapless phases correspond to spin-density-wave (SDW) and nematic phases for small and large magnetization, respectively, already identified in the zigzag Heisenberg chain with ferromagnetic NN interactions and antiferromagnetic next-nearest-neighbor (NNN) interactions [95] (see Appendix D).

\section{MANY-BODY PHASES IN TOPOLOGICAL-WAVEGUIDE QED}

\section{A. Dimerized 1D photonic bath (upper and lower band gaps)}

We now analyze the cases where $\tilde{\delta} \neq 0$. Again, we start by considering the limit $\xi \rightarrow 0$, i.e., only NN couplings. As we see, the dimerization produces VBS phases, even though there is no frustration in the system (the system is always bipartite in the NN limit, regardless of the
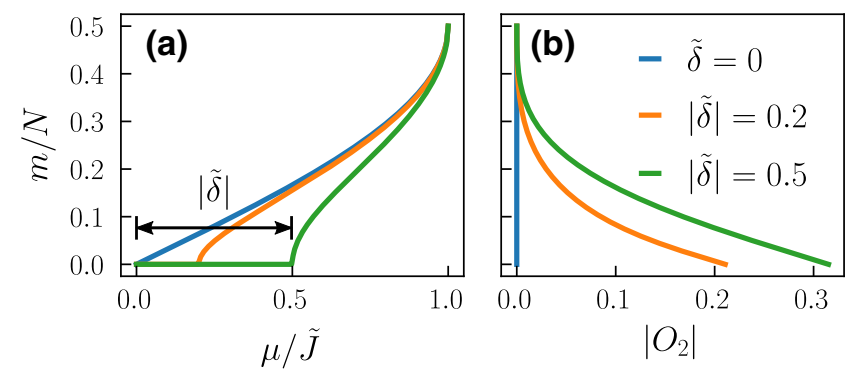

FIG. 5. The dimerized NN-XX model $(\theta=\pi / 2)$. (a) Magnetization curves for different values of the effective dimerization. (b) The dimer VBS order parameter as a function of the magnetization for several values of $|\tilde{\delta}|$ [same as in (a)].

value of $\tilde{\delta}$ ). This can be better understood in the $\mathrm{XX}$ models, $\theta= \pm \pi / 2$, which can be mapped exactly to a free fermionic SSH model by a Jordan-Wigner transformation (see Appendix E). A nonzero dimerization of the interactions opens a gap in the energy spectrum between the ground state with zero magnetization $(m=0)$ and the ground states of other magnetization sectors $(m \neq 0)$. This can be observed in the magnetization curves for those models as an initial plateau the width of which increases by increasing $|\tilde{\delta}|$ [see Fig. 5(a) (indicated by a black arrow)], meaning that the chemical potential — or external magnetic field in the spin language - has to be larger than a critical value in order to polarize the spins in the chain. Correlations become dimerized with a dimerization pattern that follows that of the underlying photonic SSH bath. In contrast to the models discussed in the previous section, the phases with nonzero magnetization also feature a nonzero value of $\left|\mathrm{O}_{2}\right|$ that decreases as the magnetization increases, as shown in Fig. 5(b).

Since the dimerized NN-XX model can be mapped to the fermionic SSH model, the topology of the former [96] can be linked straightforwardly to that of the latter. Thus, we expect two distinct symmetry-protected topological (SPT) phases at $m=0$ : one for $\tilde{\delta}>0$ (trivial SPT phase) and another for $\tilde{\delta}<0$ (nontrivial SPT phase). These SPT phases extend to other values of $\theta$ around the XX point and larger values of $\xi$, which we indicate in the phase diagrams shown in Figs. 6(a) and 9(a) with the label $\mathrm{VBS}_{2}$. Since these models can be connected adiabatically to the freefermion model without closing the gap, they all belong to the same SPT phases. Importantly, in the fermionic language, these phases for other values of $\theta \neq \pi / 2$ correspond to models of interacting fermions. Thus, we can argue that these noninteracting SPT phases are robust against some amount of interaction. More specifically, they are robust against correlated fermion hopping, which in the spin language corresponds to the addition of long-range spin interactions; and also to certain amount of fermion density-density interactions, which in the spin language corresponds to nonzero $\mathrm{ZZ}$ interactions. 

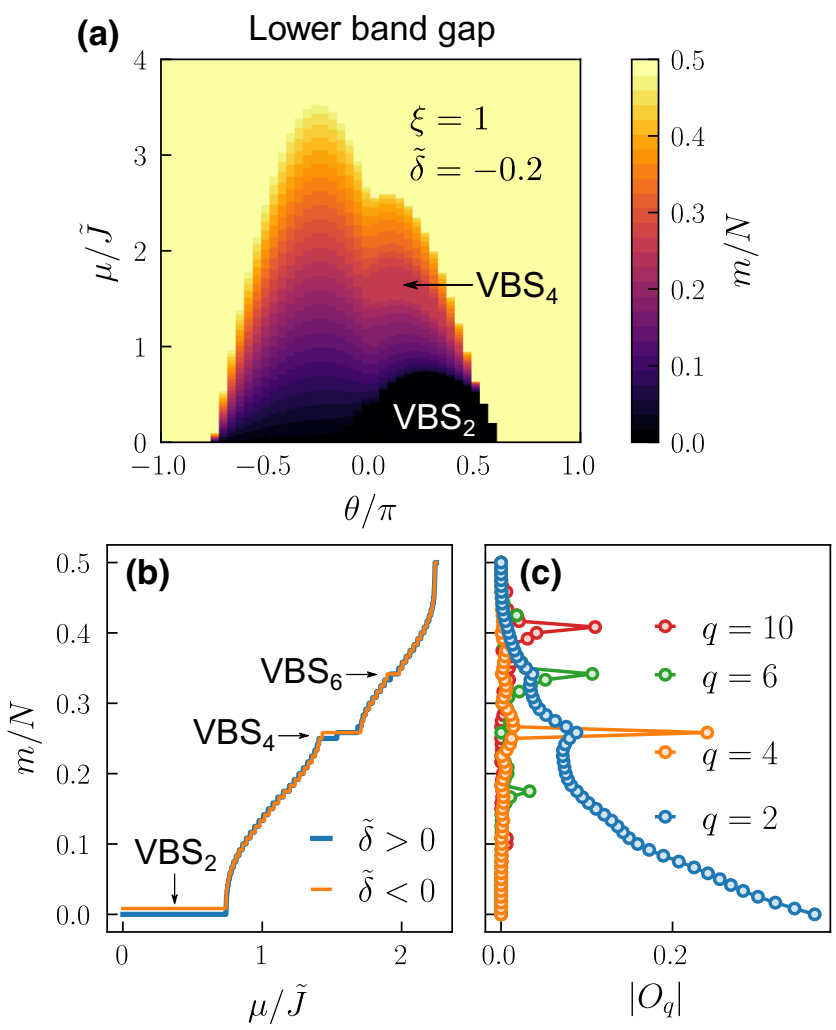

FIG. 6. The dimerized intermediate-interaction-range LBG model. (a) The ground-state magnetization as a function of the external magnetic field and the anisotropy angle for a system with $N=50$ spins. (b) The magnetization curve for a longer chain with $N=120$ for $\theta=\pi / 4$ and $\tilde{\delta}= \pm 0.2$. (c) The bondorder parameters (structure factor) for the model with $\theta=\pi / 4$ and $\tilde{\delta}=0.2$ as a function of the magnetization. In all the figures, the interaction length is set to $\xi=1$.

The distinction between the two possible SPT phases becomes apparent when considering chains with open boundary conditions (OBCs). In the nontrivial SPT phase $(\tilde{\delta}<0)$, the ground state is fourfold degenerate in the thermodynamic limit if $\mu=0$ due to the presence of two uncoupled spin-1/2 degrees of freedom located at the edges of the chain. This degeneracy and hence the presence of these edge modes are consequences of symmetry fractionalization [97]. Both features are protected by symmetries (hence the term "symmetry-protected" topological phase), in this case, time reversal and the group generated by $\pi$ rotations around two orthogonal spin axes. For $\mu \neq 0$, the model no longer possesses those symmetries. The free spins at the ends of the chain in the topological phase align with the external magnetic field, resulting in a difference of the ground-state magnetization $m(\delta<0)-m(\delta>0)=1$ for $\mu \rightarrow 0^{+}$, which can be clearly appreciated in the magnetization curves shown in Figs. 6(b) and 9(b) (compare the blue and orange curves, which have a value that differs by 1 in the initial plateau).
Formally, these different SPT phases can be distinguished by the geometric phases accumulated by the ground state when modulating periodically ("twisting") the Hamiltonian in certain specific ways. These manybody Berry phases may be quantized depending on the symmetries of the model and on the specific twist performed [68-70]. For example, for a time-reversal symmetric phase, such as the $\mathrm{VBS}_{2}$ phase, a modulation of the interaction term between any two spins $m, n \in\{1, \ldots, N\}$ of the form

$$
S_{m}^{+} S_{n}^{-}+\text {H.c. } \rightarrow e^{-i \phi} S_{m}^{+} S_{n}^{-}+\text {H.c. }
$$

as $\phi$ varies through $[0,2 \pi)$ will result in a Berry phase equal to 0 or $\pi(\bmod 2 \pi)$, depending on whether the two spins are weakly or strongly entangled, respectively [68] (see Appendix F). The many-body Berry phases computed between neighboring spins thus reveal the pattern of singlets characteristic of each SPT order. We obtain different values depending on whether the two spins belong to the same or adjacent unit cells, $\gamma_{\text {intra-inter }}$ :

$$
\left(\gamma_{\text {intra }}, \gamma_{\text {inter }}\right)=\left\{\begin{array}{ll}
(\pi, 0), & \text { for } \tilde{\delta}>0, \\
(0, \pi), & \text { for } \tilde{\delta}<0,
\end{array} \quad \bmod 2 \pi .\right.
$$

Surprisingly, for a finite interaction length, $\xi>0$, the models in the LBG develop new gapped phases at magnetizations $m / N \approx 1 / 4$ and $m / N \approx 1 / 3$. These phases correspond to the magnetization plateaus shown in Figs. 6(a) and 6 (b) for $\theta \approx \pi / 4$, labeled as $\operatorname{VBS}_{q}$, with $q=4,6$. When we compute the bond-order parameter $O_{q}$ for different periodicities $q$, shown in Fig. 6(c), we observe that in these phases $\left|O_{q}\right|$ has a maximum for $q \neq 2$, implying that they are periodic with a period involving more than one unit cell. We also observe that the first derivative of the dimer-bond-order parameter $\left|O_{2}\right|$ seems to have a discontinuity at those phases. This increased period can also be appreciated in some of the plots in Fig. 7, where we show the individual spin magnetization for chains with OBCs in the $\mathrm{VBS}_{4}$ phase. This suggests the following simplified picture of the ground state in these higher-period VBS phases: it is formed by "breaking" in a periodic fashion some of the valence bonds present in the $\mathrm{VBS}_{2}$ phase, as shown schematically in Fig. 8. This intuitive picture also explains the $(q / 2)$-fold degeneracy of the ground state in the sector with magnetization $m / N=1 / 2-1 / q$ found in chains with periodic boundary conditions (PBCs), as there are $q / 2$ different ways to break the bonds in order to achieve a phase with that magnetization.

Similarly to the $\mathrm{VBS}_{2}$ phase, these $\operatorname{VBS}_{q}(q>2)$ phases are also SPT phases that can either be topologically trivial $(\tilde{\delta}>0)$ or $\operatorname{not}(\tilde{\delta}<0)$. This is confirmed by computing the (non-Abelian) many-body Berry phases (see Appendix F), which are the same as in the $q=2$ phase, given in Eq. (25). However, in contrast to the $q=2$ phase, these SPT phases 

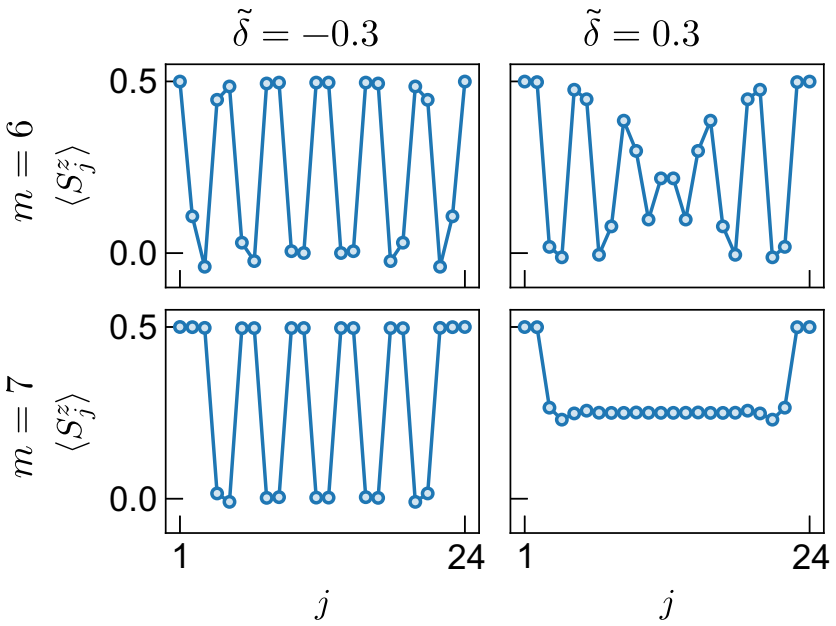

FIG. 7. The dimerized intermediate-interaction-range LBG model. The individual-spin magnetization for chains with $N=$ 24 spins with $\mathrm{OBCs}$ at the isotropic point $\theta=\pi / 4$. The upper (lower) row corresponds to phases with $m=N / 4(m=N / 4+$ 1 ), whereas the left (right) column corresponds to phases with a negative (positive) dimerization constant. The interaction length is set to $\xi=1$ in all cases. In all cases considered, the ground state is unique, except for the case with $\tilde{\delta}>0$ and $m=N / 4+1$, where it is doubly degenerate. The bulk of the chain in this case would be the one that resembles most closely the $\mathrm{VBS}_{4}$ state in chains with PBCs.

at higher magnetization are protected solely by spaceinversion symmetry. As it turns out, in this case nontrivial chains with OBCs do not feature topological edge states $[98,99]$ and the ground state of trivial chains with OBCs

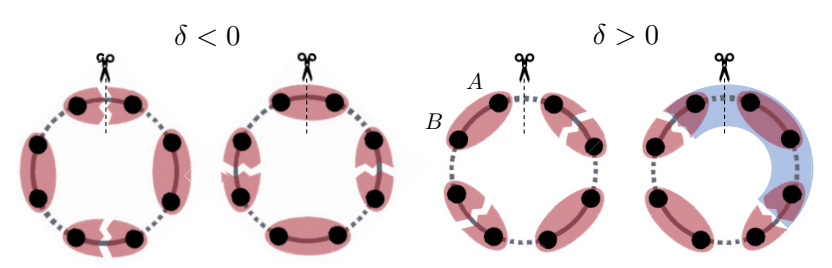

FIG. 8. A schematic representation of the $\mathrm{VBS}_{4}$ phase. The red ellipses represent valence bonds (singlets) between adjacent spins (black dots). The split ellipses represent broken valence bonds in which the two spins are fully polarized along the $z$ axis. The blue shaded area represents the space-inversion symmetric "cluster" that repeats along the chain in this VBS phase. For each distinct SPT phase (corresponding to positive or negative values of the dimerization constant $\delta$ ), there are two possible patterns of broken bonds and, hence, the ground state of chains with PBCs is twofold degenerate. If we consider chains with OBCs, we see that for $\delta<0$, any of the two patterns can be cut open still respecting inversion symmetry (the leftmost pattern is energetically more favorable for $m=N / 4$, while the other one is preferred for $m=N / 4+1)$. For $\delta>0$, by contrast, this is not possible and there is no preferred pattern of broken bonds for chains with OBCs (cf. Fig. 7). presents a twofold degeneracy only in the case with $\tilde{\delta}>0$ and $m=N / 4+1$, which is not attributed to the presence of edge modes but, rather, to a special kind of frustration related to the lack of inversion symmetry of the two possible patterns of broken bonds, as explained intuitively by the schematics shown in Fig. 8.

We remark that the appearance of these higher-period VBS phases requires the conjunction of long-range exchange and $\mathrm{ZZ}$ interactions and a nonzero dimerization. In the fermionic language, we can say that they appear due to the presence of interactions between fermions, both of the density-density and of the correlated-hopping kind.

As for the dimerized UBG model with a finite interaction length, a similar analysis follows for the $\mathrm{VBS}_{2}$ phase located around $\theta \approx-\pi / 2$ : it is topologically trivial (nontrivial) if $\tilde{\delta}>0(\tilde{\delta}<0)$. Curiously, we observe that the dimerization affects the SDW phase appearing at $\theta \approx-3 \pi / 4$ in low external magnetic fields. As shown in Fig. 9(b), for $\tilde{\delta}<0$, the magnetization changes in steps of $\Delta m=1$, instead of $\Delta m=2$, which is the case for $\tilde{\delta}>0$. This difference suggests the presence of "persistent edge modes," that is, almost free spin- $1 / 2$ degrees of freedom at the edges of the chain that align in the direction of the external magnetic field. This is further confirmed in Fig. 9(c), where we show the individual spin magnetization for chains with OBCs in the SDW phase. These topological edge states, which are present even though the system remains in a gapless phase, could be explained in terms of symmetry-enriched conformal-field theories, which have recently attracted a lot of attention $[100,101]$.

\section{B. Middle band gap}

For $\delta \neq 0$, a new gap (the MBG) appears in the energy spectrum of the bath. In this gap, all spin coupling constants $J_{n}^{\alpha \beta}$ alternate sign as a function of distance $n$. Due to its peculiar properties and physical significance, we study separately the case where the emitters are spectrally tuned to the middle of the MBG $(\Delta=0)$ and the case with $\Delta \neq 0$.

\section{Case with $\Delta=0$}

In this case, the system is fully dimerized, $\tilde{\delta}=\operatorname{sign}(\delta)$; i.e., the only nonzero coupling constants are either $J_{n}^{A B}$ or $J_{n}^{B A}$, depending on the dimerization pattern of the underlying SSH photonic bath. This implies that the system is always bipartite, even for a finite interaction length $(\xi>0)$. As a consequence, the phase diagram is always symmetric with respect to the change $\theta \rightarrow-\theta$ [see Figs. 10(a)-10(c)]. In chains with OBCs, a negative value of the dimerization constant implies the presence of two uncoupled spins at the edges, regardless of the value of the rest of the parameters. In other words, all phases for $\delta<0$ feature edge states - even when the bulk is in a gapless phase. 


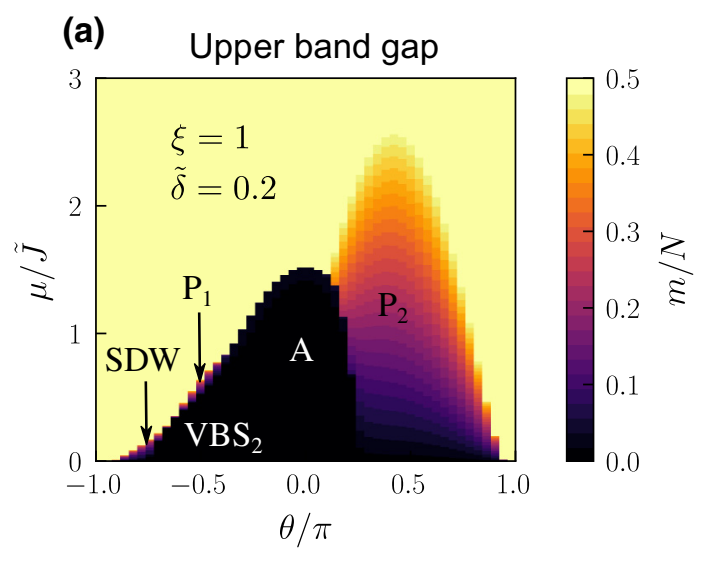

(b)
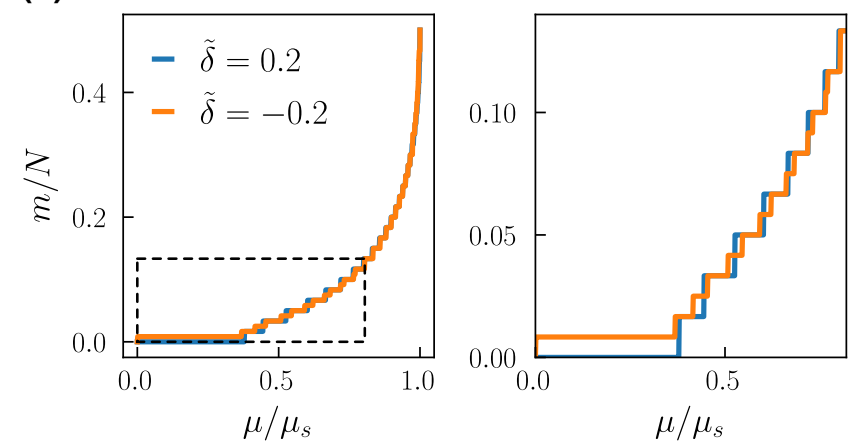

(c)

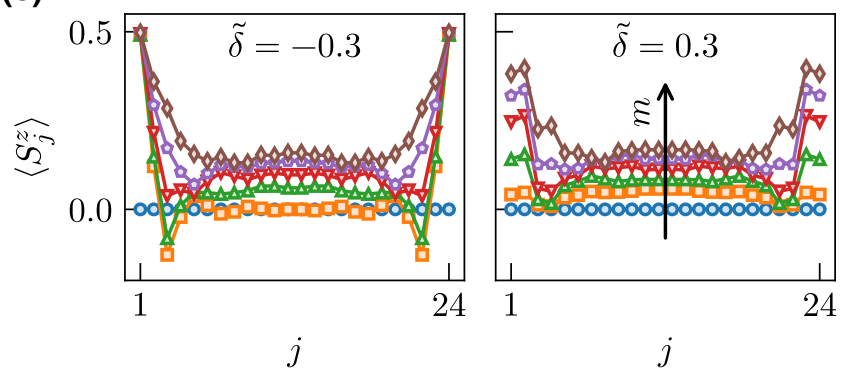

FIG. 9. The dimerized intermediate-interaction-range UBG model. (a) The ground-state magnetization as a function of the external magnetic field and the anisotropy angle for a system with $N=50$ spins. (b) The magnetization curve for a longer chain with $N=120$ for $\theta=-3 \pi / 4$ and $\tilde{\delta}= \pm 0.2$. The right-hand plot is just an enlargement of the lower magnetization range. (c) The individual-spin magnetization for a chain with $N=24$ spins and OBCs, at $\theta=-0.65 \pi$. The values correspond to the ground states of the sectors with total magnetization $0 \leq m \leq 5$. Even though for $\tilde{\delta}>0$ there is a considerable effect of the edges, in the case with $\tilde{\delta}<0$ the difference with respect to the bulk is starker, with the ending spins being fully polarized regardless of the value of the total magnetization. In all the panels, the interaction length is set to $\xi=1$.

As we do in the other regimes, let us consider first the $\mathrm{NN}$ model. In the limit $\delta \rightarrow \pm 1$, keeping $\Delta=0$, the model corresponds to a set of disconnected dimers, so its phase diagram is trivial to obtain, as one only has to consider the Hilbert space of two spins. There are just two phases:

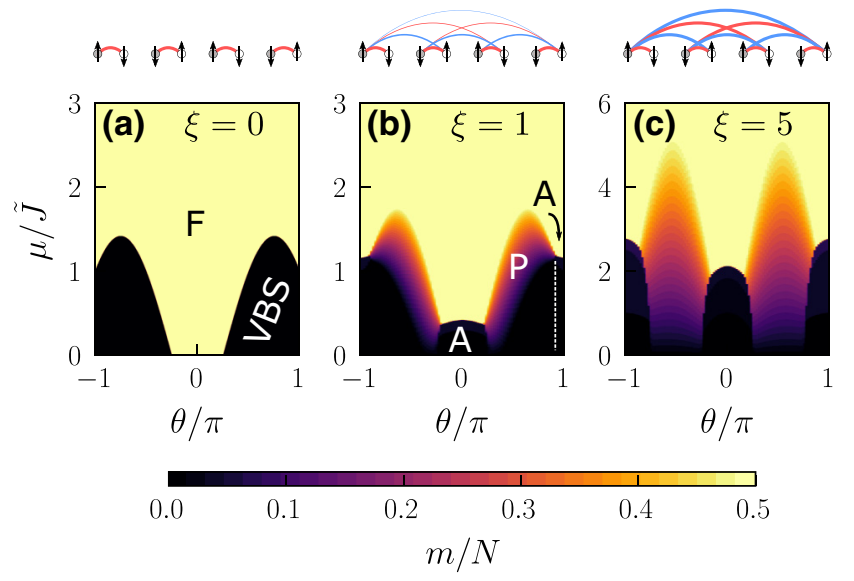

(d)

\begin{tabular}{|c|c|c|c|c|c|c|c|}
\hline 0 & $\begin{array}{l}\xi=5 \\
\nu=z\end{array}$ & 口 & $\begin{array}{l}\xi=5, \\
\nu=x\end{array}$ & $\Delta$ & $\begin{array}{l}\xi=1, \\
\nu=z\end{array}$ & $\diamond$ & $\begin{array}{l}\xi=1, \\
\nu=x\end{array}$ \\
\hline
\end{tabular}
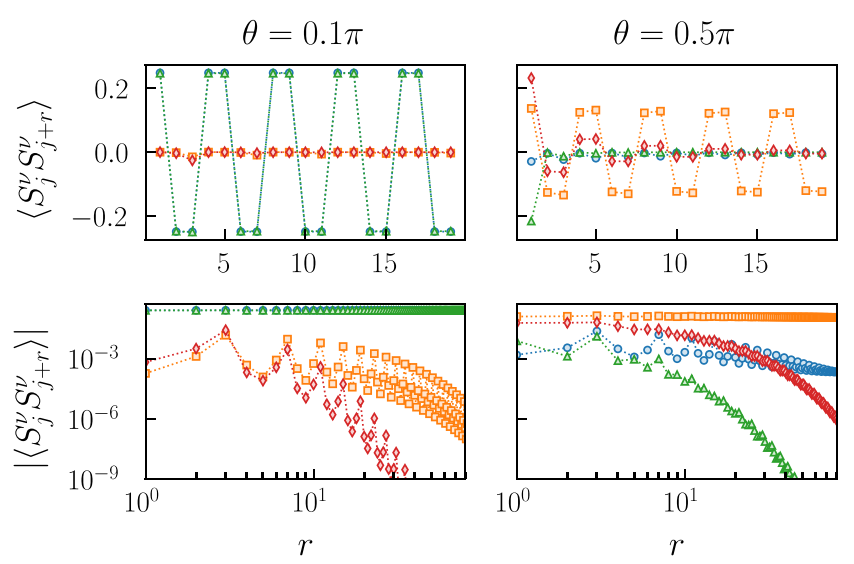

FIG. 10. The fully dimerized $(\Delta=0, \tilde{\delta}= \pm 1)$ MBG model. (a)-(c) Ground-state-magnetization phase diagrams for chains with $N=60$ spins and OBCs, for different interaction lengths. The stability region of different phases is indicated with letters: F, ferromagnetic; A, antiferromagnetic; P, planar; VBS, valencebond solid. (d) Correlations in the phase with zero magnetization $m=0$ for a chain with $N=120$ at $\theta=0.1 \pi$ (antiferromagnetic phase) and at $\theta=\pi / 2$ (The same data are shown in both plots, simply changing the scale of the axes; the legend refers to both of them.

a phase with zero magnetization where the ground state is a product of singlets, and a ferromagnetic phase [see Fig. 10(a)]. Note that a product of singlets is nothing but a valence-bond solid phase. As we increase the interaction length, we still observe VBS phases for small values of the chemical potential and values of the anisotropy angle $\theta \approx \pm \pi / 2$. In addition, new planar and antiferromagnetic phases appear in the phase diagram [see Fig. 10(b)]. These phases are similar to those found in the NN-XXZ Heisenberg model, but with a doubled period, which is a consequence of the alternating sign of the interactions. This doubled period can be appreciated in Fig. 10(d), where we show the correlations in the ground state for the phase 
with $m=0$ and two different anisotropy angles, $\theta=0.1 \pi$ and $\theta=\pi / 2$. When we increase the range of interactions, the VBS phase becomes less stable and eventually disappears, leading to a gapless phase featuring a double Néel order similar to that of the antiferromagnetic phases but this time in the $x-y$ plane, as shown in Fig. 10(d) (case with $\xi=5$ ). The limit of infinite interactions can be approached as $\delta \rightarrow \pm 0^{+}$, keeping $\Delta=0$. This infinite-range model cannot be studied using collective spin operators, since spins in one sublattice only interact with spins in the other sublattice to their right or left [as shown in the schematic depiction of the system at the top of Fig. 10(c)]. Nonetheless, we can get an idea of its phase diagram by looking at systems with large but finite $\xi$, such as the one shown in Fig. 10(c). As we can observe in that figure (and the plot of the correlations below), in the limit $\mu \rightarrow 0$ the ground state always has $m=0$ and there are only two distinct phases featuring constant correlations (independent of distance) either in the $z$ axis (antiferromagnetic phase) or in the $x-y$ plane (infinite-range planar phase).

\section{Case with $\Delta \neq 0$}

For systems in the MBG with $\Delta \neq 0$, the model is no longer bipartite - there are nonzero couplings connecting spins within the same sublattice - which makes the phase diagram asymmetric with respect to the change $\theta \rightarrow-\theta$; for an example of a phase diagram in this regime, see Fig. 11(a).

In this case, there is no model with $\mathrm{NN}$ interactions, i.e., it is not possible to reach $\xi \rightarrow 0$ keeping $\Delta \neq 0$ in the MBG. However, there are two possible infinite-range models that occur for $\Delta \rightarrow \pm 2|\delta| J$ (inner band edges), if $\delta \neq 0$. The coupling constants in these models are $J_{n}^{A B}=$ $\operatorname{sign}(\delta)(-1)^{n} \tilde{J}, J_{n}^{B A}=-\operatorname{sign}(\delta)(-1)^{n-1} \tilde{J}$ and $J_{n}^{A A / B B}=$ $\operatorname{sign}(\Delta)(-1)^{n-1} \tilde{J}$. As it turns out, the Hamiltonian can be written using collective spin operators and it is the same (or the same with opposite sign) as the one shown in Eq. (21) for $\Delta \rightarrow 2|\delta| J(\Delta \rightarrow-2|\delta| J)$. Consequently, the phase diagram is exactly the same as the one shown in Fig. 3(b) (or the same, shifting $\theta \rightarrow \theta+\pi$ ). The definition of the collective operators is either $S_{A}^{v}=\sum_{n}\left(S_{2 n, A}^{v}+S_{2 n, B}^{v}\right)$ and $S_{B}^{v}=\sum_{n}\left(S_{2 n+1, A}^{v}+S_{2 n+1, B}^{v}\right)$ or $S_{A}^{v}=\sum_{n}\left(S_{2 n, A}^{v}+S_{2 n+1, B}^{\nu}\right)$ and $S_{B}^{v}=\sum_{n}\left(S_{2 n+1, A}^{v}+S_{2 n, B}^{v}\right)$, depending on $\operatorname{sign}(\delta)$, which reflects the facts that the period of the ground state is doubled due to the alternating sign of the interactions and that changing the sign of the dimerization parameter is equivalent to a redefinition of the unit cell of the model.

For intermediate-interaction ranges, we find phases similar to those appearing for $\Delta=0$; apart from the asymmetry, the phase diagram shown in Fig. 11(a) is similar to the one shown in Fig. 10(c). Surprisingly, for some anisotropy angles, the edge states of the gapless phases still survive when the ending spins are coupled to the rest of

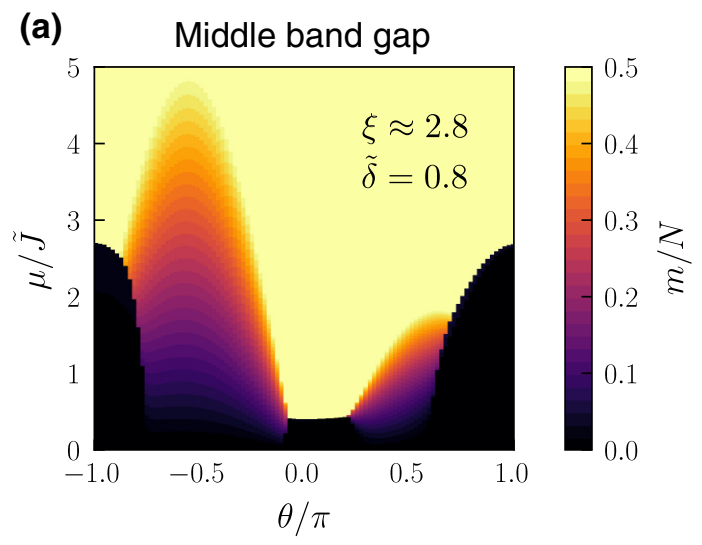

(b)
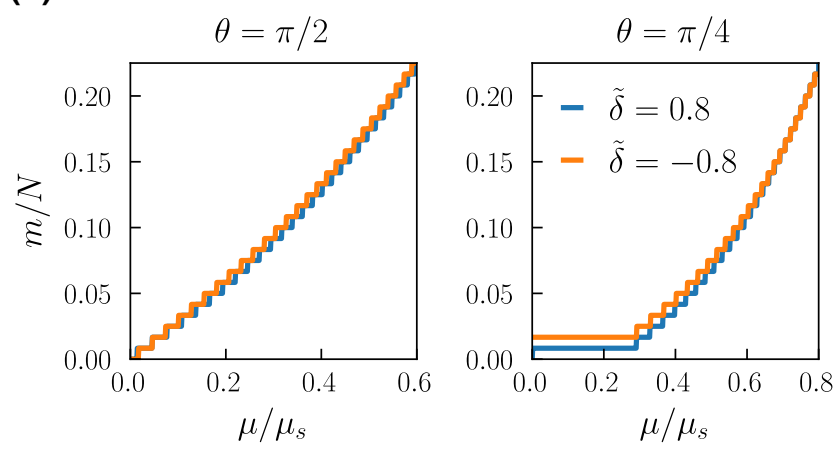

FIG. 11. The partially dimerized $(\Delta \neq 0, \quad|\tilde{\delta}|<1)$ intermediate-interaction-range MBG model. (a) Groundstate-magnetization phase diagrams for a system with $N=60$ spins and OBCs. (b) Magnetization curves for a longer chain with $N=120$ spins and the same parameters.

the chain, as is suggested by the difference in the magnetization curves for $\tilde{\delta} \gtrless 0$ shown in Fig. 11(b) (case with $\theta=\pi / 4)$.

\section{EXPERIMENTAL CONSIDERATIONS}

In this section, we discuss several aspects related to the experimental realization of the physics described throughout this paper. First, in Sec. V A, we briefly review different waveguide-QED platforms, commenting on their potential to observe the different models explored throughout this paper. Then, in Sec. VB, we explicitly show how in the most accessible model in experiments $(\theta=\pi / 2)$, even for finite systems, one will be able to observe signatures of a topological phase transition just by controlling the frequency of the emitters. Finally, in Sec. VC, we show how adiabatic protocols can be used to access the different many-body phases discussed in this work.

\section{A. Potential waveguide-QED simulators}

Nowadays, there is a plethora of systems where waveguide-QED physics can be engineered to certain extent. Some of the most relevant ones are as follows: 
1. Cold atoms trapped near nanophotonic structures [57]. This is the system where the original proposal of a waveguide-QED simulator for spin models appeared $[54,79]$. Compared to other platforms, their potential is that all emitters are equal and have a complex level structure, with multiple optical transitions, which can be harnessed to engineer XXZ models, e.g., by stroboscopic means [56] (see Appendix B). The first experiments have already appeared $[36,37,39,40]$, although the main challenge remains still to place the atoms in a controlled way near the surface. However, current advances in novel trapping techniques [102-106] foresee nearfuture control of this setup.

2. Solid-state emitters in nanophotonic structures. Solid-state emitters, like quantum dots [46] or vacancy centers [47], facilitate the integration at the nanophotonic structure. However, this comes at the price of interacting with the phononic environment and, importantly, the inhomogeneous broadening of emitters. However, the latter can be compensated with clever driving techniques [107] or the use of electro-optic modulators [108]. The most accessible models with these systems are the XX limits $(\theta=$ $\pi / 2$ ), although the advances in controlling Ramanassisted processes in these systems $[109,110]$ might enable the exploration of more general spin models [56].

3. Circuit $Q E D$. As shown in recent experiments [49-52], it is possible to emulate waveguide-QED physics by coupling superconducting qubits to coupled microwave resonator arrays. This system enables single-qubit addressing and readout, as well as time-dependent control of their couplings. However, since superconducting qubits generally do not feature an internal level strucutre, this platform is mostly restricted to XX-type models.

4. Cold atoms in state-dependent optical lattices. Another potential platform to obtain waveguideQED physics consists in replacing the role of the photons by matter waves. This has originally been proposed in Ref. [77] and has recently been implemented with rubidium atoms in Ref. [53]. Although coupling is not completely local, it allows for a very versatile control of light-matter interactions, which can be controlled through Raman-assisted transitions. By construction, the available models will be of the XX type.

\section{B. Exploring a topological phase transition in a small experimental system}

As mentioned above, the easier model to access within waveguide-QED simulators would be of the XX type (i.e., $\theta=\pi / 2$ ). This appears when emitters interact via (a)

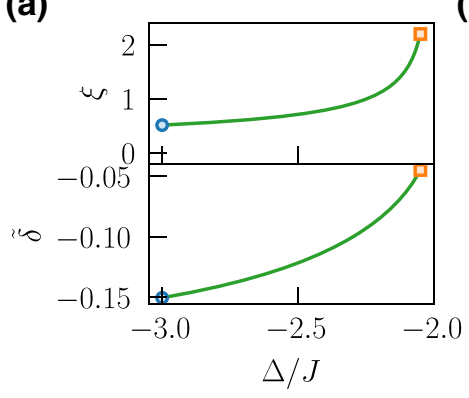

(b)

(c)
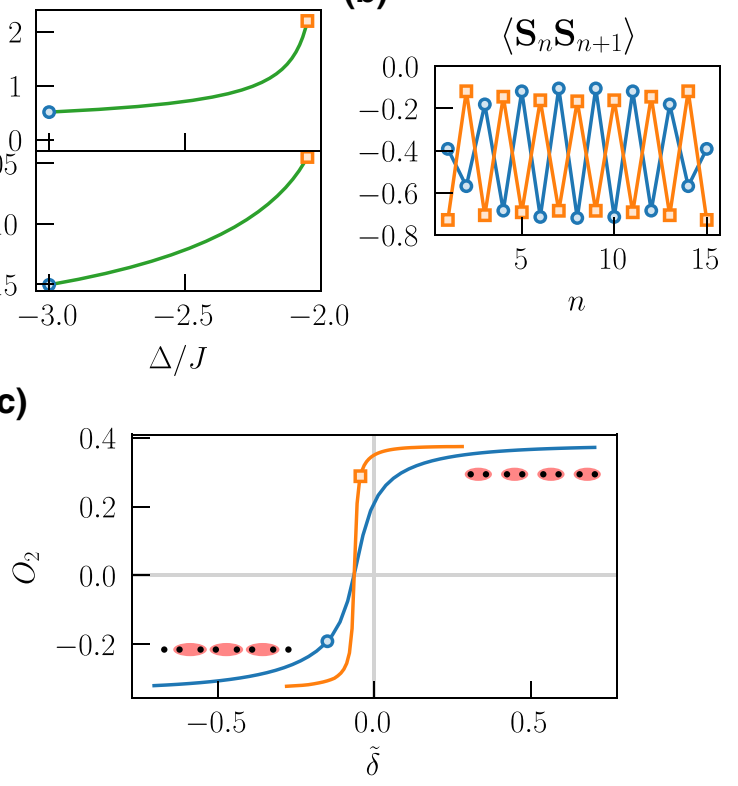

FIG. 12. (a) The dependence of the interaction range and the effective dimerization constant on the emitter detuning, for a system with $\delta=-0.2$. (b) Ground-state nearest-neighbor bond correlations for a chain with $N=16$ emitters and $\mathrm{OBCs}$, in the $m=0$ sector of the LBG-XX model $(\theta=\pi / 2)$, for two different values of the emitter detuning (marked with a blue dot and an orange square in the other panels). Large (negative) values correspond to neighboring spins that are approximately forming a singlet. (c) The dimer bond-order parameter for the same system as in (b), as a function of the effective dimerization constant.

a single-dipole transition with the waveguide modes, as can occur in all implementations. In this section, we show that by tuning the frequency of the emitters, one can tune the effective parameters of the model, as demonstrated in Fig. 12(a). In this manner, it is possible to observe the smoking gun of a topological phase transition occurring in the system, that is, a change of the dimerization pattern of the array, as shown in Fig. 12(b), for a small chain with OBCs.

Strictly speaking, topological invariants are bulk quantities, so they are not well defined for small systems. Furthermore, there are no proper phase transitions for small systems, since, typically, the ground state remains always gapped when tuning the parameters of the Hamiltonian. Still, one can define sharp transitions between different phases in finite systems according to the value of some observable that characterizes the different phases. For example, for noninteracting systems, one can define a sharp topological phase transition according to the presence or absence of single-particle edge states. The critical values of the parameters where these edge states appear are different (but close) to the critical values where phase transitions occur in the thermodynamic limit [111]. In our model in the LBG, there is a topological phase transition 
in the $m=0$ sector between two phases with opposite dimerization [two VBS phases with a different pattern of singlets; see the inset of Fig. 12(c)] that in the thermodynamic limit occurs at $\tilde{\delta}=\delta=0$. The different dimerization pattern of the ground state is captured by a change in sign of the dimer bond-order parameter $\mathrm{O}_{2}$, defined in Eq. (23). For small chains, the change in sign of $\mathrm{O}_{2}$ does not occur at $\tilde{\delta}_{c}=0$ but for $\tilde{\delta}_{c}<0$, as shown in Fig. 12(c). Furthermore, we observe that the value of $\mathrm{O}_{2}$ changes more abruptly across the transition point as the interaction length increases (compare the orange curve with the blue one). Interestingly, the effective dimerization constant of the spin model, $\tilde{\delta}$, also changes as the detuning $\Delta$ is varied, although its sign is determined by the sign of the dimerization constant of the bath $\delta$. In principle, one would not expect a phase transition to occur if the sign does not change; however, since in finite systems the critical point occurs for a different value $\tilde{\delta}_{c} \neq 0$, it is still possible to observe a phase transition only by varying the detuning of the emitters.

\section{Adiabatic preparation}

Another important aspect of analog quantum simulators is how to reach the different many-body phases that can be explored within the simulators. A standard approach is to use adiabatic protocols [112], in which one initially prepares the simulator in an easy-to-prepare state with the interactions switched off and then turns on the interactions such that the ground state of the systems evolves adiabatically until the desired state. In this section, we show precisely how one could employ an adiabatic protocol to prepare one of the phases of interest of our model. For that, we assume a setup where interactions in the $x-y$ plane and along the $z$ axis can be tuned independently, i.e., one can simulate Hamiltonians of the form $H=\tilde{J}\left(a H_{z}+b H_{x y}\right)$, with $-1 \leq a, b \leq 1$. Here,

$$
\begin{aligned}
H_{z} & =\frac{1}{\tilde{J}} \sum_{j, n>0} \sum_{\alpha, \beta} J_{n}^{\alpha \beta} S_{j, \alpha}^{z} S_{j+n, \beta}^{z}, \\
H_{x y} & =\frac{1}{\tilde{J}} \sum_{j, n>0} \sum_{\alpha, \beta} \frac{J_{n}^{\alpha \beta}}{2}\left(S_{j, \alpha}^{+} S_{j+n, \beta}^{-}+\text {H.c. }\right)
\end{aligned}
$$

are adimensional Hamiltonians and $\tilde{J}$ denotes the largest coupling constant that can be produced in the experiment. Additionally, we assume that we can prepare any product state of up-down spins easily.

For the sake of concreteness, we focus on preparing the ground state of the $\mathrm{VBS}_{4}$ phase in a system of $N=8$ emitters (spins). Note that in such small systems, there are no proper phase transitions and we may only observe some of the features of the phases in which we are interested. For example, in the case of the $\mathrm{VBS}_{4}$ phase, we would like to observe the formation of enlarged unit cells consisting of

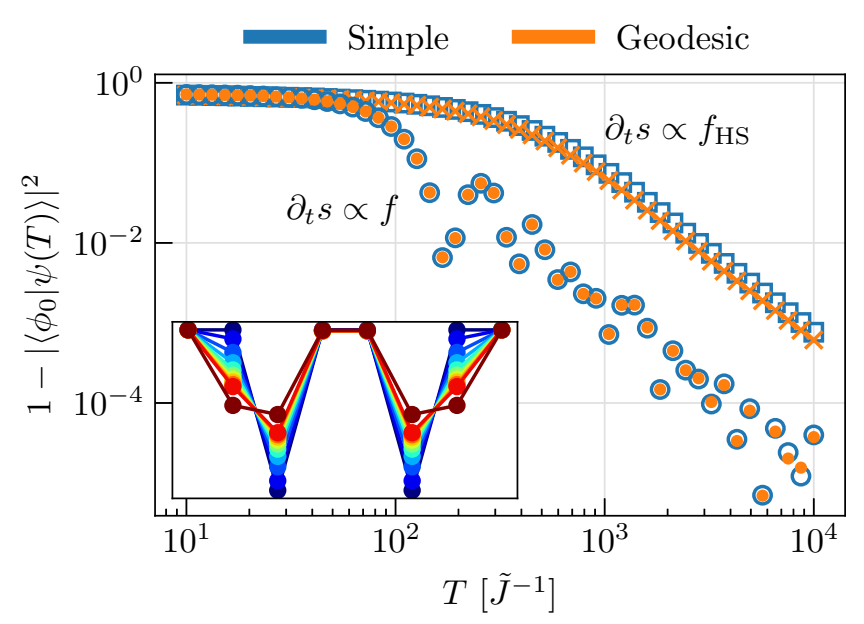

FIG. 13. The infidelity of the final state $|\psi(T)\rangle$ with respect to the target ground state $\left|\phi_{0}\right\rangle$ as a function of the preparation time $T$ for different paths in parameter space and different schedule functions. The inset shows the evolution of the individual spin magnetization at different stages of the protocol (from blue in the beginning to red toward the end). The parameters of the model are $N=8, N_{\uparrow}=6, \tilde{\delta}=-0.2$, and $\xi=2.0$; the emitters are tuned to the lower band gap.

four spins, with the two middle spins forming a singlet and the two outer spins polarized "up" (see the inset in Fig. 13).

Since any product state of up-down spins is an eigenstate of $H_{z}$, our starting Hamiltonian is $H(0) \propto H_{z}$. Our target Hamiltonian is $H(T) \propto H_{z}+H_{x y}$ [the Hamiltonian given in Eq. (1) in the Heisenberg limit, $\theta=\pi / 4]$. To compare different protocols and simplify the discussion, we reparametrize the time-dependent Hamiltonian in terms of a (bijective) "schedule function" $s:[0, T] \rightarrow[0,1]$, $H(t)=H(s(t))$. Ideally, we would like to employ an optimal modulation $[a(s), b(s)]$ that transforms the initial state into the target state within a given fidelity in the shortest amount of time. One possibility to design the protocol is to employ the geometrical approach developed in Ref. [113]. There, it has been shown that for a schedule function satisfying $\partial_{t} s \propto \Delta^{2}(s) /\left\|\partial_{s} H\right\|_{\mathrm{HS}} \equiv f_{\mathrm{HS}}(s)$, where $\Delta(s)$ is the energy gap between the ground and first excited states of $H(s)$ and $\|\cdot\|_{\mathrm{HS}}$ denotes the Hilbert-Schmidt norm, finding the optimal path in parameter space is equivalent to finding a shortest-distance geodesic in a Riemannian manifold, a problem that can be solved numerically using standard techniques $[114,115]$. In practice, for the problem at hand, we find that a simple path of the form $a(s)=1, b(s)=s$ is very close to the actual geodesic and yields very similar fidelities (see Fig. 13). Interestingly, we can improve the preparation time if we consider instead a schedule function satisfying $\partial_{t} s \propto \min _{n>0} \Delta_{n}^{2}(s) /\left|\left\langle\phi_{n}(s)\left|\partial_{s} H\right| \phi_{0}(s)\right\rangle\right| \equiv$ $f(s)$, where $\Delta_{n}(s)$ denotes the energy gap between the instantaneous ground state $\left|\phi_{0}(s)\right\rangle$ and the $n$th instantaneous excited state $\left|\phi_{n}(s)\right\rangle$. 
So far, we have considered the fidelity of the target state only considering the imperfections introduced by the adiabatic protocols, in which the slower the process is made, the better is the fidelity obtained (see Fig. 13). In real-life experiments, however, there are other decoherence sources affecting the fidelity of the final state. For concreteness, we consider one of the simplest but also more relevant error sources that can occur in waveguide-QED setups, that is, the possibility of losing emitter excitations at rates $\gamma$. The evolution in the presence of this kind of loss can be modeled through a Markovian master equation of the form

$$
\partial_{t} \rho=-i[H(t), \rho]+\gamma \sum_{j} \mathcal{D}_{j}[\rho]
$$

where $\mathcal{D}_{j}[\rho] \equiv S_{j}^{-} \rho S_{j}^{+}-\left\{S_{j}^{+} S_{j}^{-}, \rho\right\} / 2$ and $\rho$ is the reduced density matrix of the emitters. For these dynamics, it can be shown that the fidelity with respect to the target ground state is $\mathcal{F}_{\gamma}=e^{-\gamma N_{\text {exc }} T} \mathcal{F}$, where $\mathcal{F}$ would be the fidelity if there were no losses and $N_{\text {exc }}$ is the number of excitations in the target state. For example, for the simple-path protocol with $\partial_{t} s \propto f(s)$, we see that values of the decay rate $\gamma \lesssim 10^{-4} \tilde{J}$ allow us to produce the target state with an $80 \%$ fidelity or more.

We remark that the preparation time is limited essentially by the minimum gap of $H(s)$ throughout the protocol, which in the case analyzed occurs at the beginning, when $H(s) \simeq H_{z}$. The times shown in Fig. 13 could be improved if one could enlarge the gap between the initial state and the rest of the states. This can be done, for example, by implementing local magnetic fields $\sum_{j} h_{j} S_{j}^{z}$, independently for each emitter with the appropriate pattern, and then turning them off toward the end of the protocol.

\section{SUMMARY OF THE RESULTS}

Due to the wealth of different regimes and phases we cover throughout the paper, in this section we find it useful for the reader to summarize the main results in each situation. For the undimerized (standard-) waveguide simulator, there are two possible band-gap regions:

(a) For the LBG, the spin interactions are long range and with the same sign. This leads to physics that is qualitatively very similar to that found in other analog simulators with power-law interactions, e.g., trapped ions [76] [see Fig. 4(a)]. In particular, we observe a shrinking of the antiferromagnetic phase of the NN model, a Devil's staircase of phases with different magnetizations around the classical limit $(\theta \approx 0)$, and a spontaneously dimerized phase at zero magnetization for $\theta \approx \pi / 4$.

(b) For the UBG, the combination of the staggered sign of the interactions with their long-range nature leads to a qualitatively different diagram [see Fig. 4(b)]. In particular, we find that the antiferromagnetic phase extends over a larger area of the phase diagram and new gapless phases develop for $\theta \approx-3 \pi / 4$, such as the spin-density-wave and nematic phases that also appear in zigzag Heisenberg chains [95].

For the topological (dimerized) waveguide, all the spin interactions inherit the dimerization of the underlying photonic bath. However, one can still find very different phases depending on which of the three different band gaps the emitters are in resonance with:

(a) In the LBG, the main effect of the dimerization is the appearance of new gapped phases, which are absent in the NN model, at certain nonzero magnetization values [see Figs. 6(a) and 6(b)]. They display large magnetic orderings, as corroborated by the calculation of the bond-order parameter $O_{p}$ [see Fig. 6(c)]. We further understand these phases by connecting them to the equivalent fermionic model and studying the quantization of their many-body Berry phases [68-70], which enables us to further distinguish different SPT phases in them, depending on the sign of the dimerization constant. These new SPT phases are due to the unique dimerized longrange nature of the interactions appearing in such topological-waveguide setups.

(b) In the UBG regime, the dimerization affects the VBS phase around $\theta=-3 \pi / 4$ at zero magnetization, giving rise to different SPT phases depending on the sign of $\tilde{\delta}$. It also affects the spin-density wave regime, as the system shows different magnetization steps as $\mu$ increases for $\tilde{\delta} \lessgtr 0$, which can be attributed to the appearance of persistent edge states.

(c) In the MBG regime for exactly $\Delta=0$, the spin interactions are fully dimerized. This has two consequences: (i) it leads to the presence of uncoupled spins at the edges for the phases with $\tilde{\delta}<0$, regardless of the value of the rest of the parameters - even in the gapless phases; and (ii) the model displays VBS, planar, and antiferromagnetic phases reminiscent of the NN model but with a double periodicity (see Fig. 10). When $\Delta \neq 0$, the interactions are not fully dimerized but we find qualitatively similar phases as in the $\Delta=0$ case, including the appearance of edge states in the gapless phases (persistent edge modes).

\section{CONCLUSIONS}

To sum up, we characterize the emergent many-body phases of a general class of spin models that can be simulated using quantum emitters subject to waveguidemediated interactions. First, we study the effect of the 
tunable, long-range, and possibly alternating-sign interactions that appear in standard waveguides. The interplay of all these features gives rise to phases different from those of the typical nearest-neighbor models, due to the larger frustration of the system. In addition, we consider the impact of the dimerized interactions appearing in topological waveguides. The latter lead to the appearance of symmetryprotected topological phases and, if they are also long range, give rise to symmetry-protected topological phases with large magnetic orderings (larger than the period of the Hamiltonian). In all cases, we explain the experimental observables that can be measured to distinguish these phases based on either single-spin measurements (individual-spin magnetization) or two-spin correlations (bond-order parameter). Finally, we also show how the most interesting phases can be reached through adiabatic protocols and analyze their fidelity in terms of the most typical error source. Overall, we believe that our work uncovers the potential of waveguide-QED setups as ana$\log$ quantum spin simulators and can become the basis for future experiments on the subject. An interesting outlook would be to study the modification of these phase diagrams when the topological waveguides have larger winding numbers, since the shape of the resulting waveguidemediated interactions can differ significantly [116].

\section{ACKNOWLEDGMENTS}

We thank Yasuhiro Hatsugai and Daniel GonzálezCuadra for enlightening discussions. M.B. acknowledges support from the ERC Advanced Grant QENOCOBA under the European Union (EU) Horizon 2020 program (Grant Agreement No. 742102), G.P. acknowledges support from the Spanish Ministry of Science and Innovation through Grants No. MAT2017-86717-P and No. PID2020117787GB-100, and A.G.-T. acknowledges support from the Spanish MCIU/AEI/FEDER (EU) project through Grant No. PGC2018-094792-B-100 and from the Proyecto Sinérgico CAM 2020 through Grant No. Y2020/TCS-6545 [Nanophotonics for Quantum Computation (Nanoquco)Comunidad de Madrid (CM)]. We all acknowledge support from the CSIC Research Platform on Quantum Technologies PTI-001.

\section{APPENDIX A: COUPLING CONSTANTS}

In this appendix, we review how the basic dipole-dipole interactions can be obtained in systems of two-level emitters coupled to a bosonic bath and we examine some of the methods that can be employed to produce more exotic effective spin interactions.

\section{Dipole-dipole (exchange) interactions}

We start by considering the case of emitters with a single-dipole transition coupled to a 1D SSH photonic bath within the rotating-wave approximation. The total Hamiltonian, describing both the emitters and the bath, reads $H=H_{S}+H_{B}+H_{I}$, with

$$
\begin{aligned}
& H_{S}=\Delta \sum_{n, \alpha} S_{n, \alpha}^{+} S_{n, \alpha}^{-}, \\
& H_{B}=-J \sum_{j}\left[(1+\delta) a_{j}^{\dagger} b_{j}+(1-\delta) a_{j+1}^{\dagger} b_{j}+\text { H.c. }\right] \\
& H_{I}=g \sum_{n, \alpha}\left(S_{n, \alpha}^{+} \alpha_{n}+\text { H.c. }\right)
\end{aligned}
$$

The operators $S_{n, \alpha}^{ \pm}$are the same as the ones introduced in the main text, while $a_{j}^{\dagger}, a_{j}\left(b_{j}, b_{j}^{\dagger}\right)$ are the bosonic creation and annihilation operators for the $A(B)$ mode in the $j$ th unit cell of the bath. The spectrum of the bath is shown in Fig. 1(b). It consists of two bands spanning the ranges $\left[\omega_{1}, \omega_{2}\right]$ and $\left[\omega_{3}, \omega_{4}\right]$, where the band-edge frequencies are $\omega_{1}=-2 J, \omega_{2}=-2 J|\delta|, \omega_{3}=2 J|\delta|$ and $\omega_{4}=2 J$. In the Markovian regime, i.e., when $g \ll \Delta_{\text {edge }}$ $\left(\Delta_{\text {edge }} \equiv \min _{j}\left|\Delta-\omega_{j}\right|\right)$, one can trace out the bath degrees of freedom, obtaining an effective description of the emitters in terms of a master equation, $\dot{\rho}=-i\left[H_{\text {eff }}, \rho\right]+\mathcal{D}[\rho]$ $[75,117]$. Here, $\rho$ denotes the reduced density matrix of the emitters. It contains a coherent part, dictated by an effective Hamiltonian

$$
H_{\mathrm{eff}}=H_{S}+\sum_{m, n} \sum_{\alpha, \beta} J_{m n}^{\alpha \beta} S_{m, \alpha}^{+} S_{n, \beta}^{-},
$$

and a dissipative part, given by

$$
\mathcal{D}[\rho]=\sum_{m, n} \sum_{\alpha, \beta} \frac{\Gamma_{m n}^{\alpha \beta}}{2}\left[2 S_{n, \beta}^{-} \rho S_{m, \alpha}^{+}-\left(S_{m, \alpha}^{+} S_{n, \beta}^{-}, \rho\right)\right] .
$$

For time-reversal-symmetric baths such as the one considered in this work, the effective interactions and collective decay rates are given by the real and imaginary parts of the collective self-energies, $\Sigma_{m n}^{\alpha \beta}\left(\Delta+i 0^{+}\right)=J_{m n}^{\alpha \beta}-i \Gamma_{m n}^{\alpha \beta} / 2$. For values of $\Delta$ in the band gaps, the decay rates vanish, $\Gamma_{m n}^{\alpha \beta}=0$, such that the emitter dynamics are governed by an XX-type spin Hamiltonian, given in Eq. (A4).

The collective self-energies have been obtained analytically in a recent work [58]. For convenience, we rewrite them here. First, we define the following functions of the complex variable $z: r(z)=\sqrt{\prod_{j}\left(z-\omega_{j}\right)}$ and

$$
y_{ \pm}(z)=\frac{z^{2}-2 J^{2}\left(1+\delta^{2}\right) \pm r(z)}{2 J^{2}\left(1-\delta^{2}\right)} \text {. }
$$

Being a translationally invariant bath, the collective selfenergies only depend on the distance between the emitters 
(but may differ depending on the sublattice to which they are coupled). Thus, we define $\Sigma_{n}^{\alpha \beta} \equiv \Sigma_{m, n+m}^{\alpha \beta}$, which are given by

$$
\begin{aligned}
& \Sigma_{n}^{A A}(z)=\mp \frac{g^{2} z y_{ \pm}{ }^{|n|}}{r(z)}, \\
& \Sigma_{n}^{A B}(z)= \pm \frac{g^{2} J\left[(1+\delta) y_{ \pm}{ }^{|n|}+(1-\delta) y_{ \pm}^{|n+1|}\right]}{r(z)},
\end{aligned}
$$

where the upper (lower) sign has to be chosen if $|\operatorname{Re}(z)|<$ $2 J|\delta|(|\operatorname{Re}(z)|>2 J)$. Furthermore, $\Sigma_{n}^{B B}(z)=\Sigma_{n}^{A A}(z)$, and $\Sigma_{n}^{B A}(z)=\Sigma_{-n}^{A B}(z)$ for $n \geq 1$. From these expressions, one can readily compute $J_{n}^{\alpha \beta}=\operatorname{Re}\left[\Sigma_{n}^{\alpha \beta}\left(\Delta+i 0^{+}\right)\right]$. We can rewrite the coupling constants in a more transparent form [see Eqs. (4)-(9)], defining an interaction length as $\xi=$ $-1 / \log \left|y_{ \pm}\right|$; an effective dimerization constant

$$
\begin{aligned}
\tilde{\delta} & \equiv \frac{\left|J_{n}^{A B}\right|-\left|J_{n+1}^{B A}\right|}{\left|J_{n}^{A B}\right|+\left|J_{n+1}^{B A}\right|} \\
& =\frac{1-e^{-1 / \xi}}{1+e^{-1 / \xi}} \times \begin{cases}\delta^{-1}, & \text { if }|\Delta|<2 J|\delta|, \\
\delta, & \text { if }|\Delta|>2 J,\end{cases}
\end{aligned}
$$

which corresponds to the dimerization of the waveguidemediated spin interactions; and the ratio

$$
\begin{aligned}
\eta & \equiv \frac{2\left|J_{n+1}^{A A}\right|}{\left|J_{n}^{A B}\right|+\left|J_{n+1}^{B A}\right|} \\
& =\frac{|\Delta| e^{-1 / \xi}}{J\left(1+e^{-1 / \xi}\right)} \times \begin{cases}|\delta|^{-1}, & \text { if }|\Delta|<2 J|\delta|, \\
1, & \text { if }|\Delta|>2 J .\end{cases}
\end{aligned}
$$

Note that $\tilde{\delta}$ and $\delta$ both have the same sign, so the dimerization pattern of the effective spin model follows that of the underlying bath. Also, from Eq. (A9), we can see that the pairs $(\xi, \tilde{\delta})$ such that $|\tilde{\delta}|<\left(1-e^{-1 / \xi}\right) /\left(1+e^{-1 / \xi}\right)$ are possible only in the outer band gaps, while the pairs satisfying $|\tilde{\delta}|>\left(1-e^{-1 / \xi}\right) /\left(1+e^{-1 / \xi}\right)$ are possible only in the middle band gap. Figure 14 summarizes at a glance the possible values of the parameters for the different models.

The global strength of the spin interactions can be computed as

$$
\tilde{J}=\frac{g^{2} J\left(1+e^{-1 / \xi}\right)}{r(\Delta)} \times \begin{cases}|\delta|, & \text { if }|\Delta|<2 J|\delta|, \\ 1, & \text { if }|\Delta|>2 J\end{cases}
$$

From this expression, we obtain the scaling $\tilde{J} \sim$ $g^{2} / \sqrt{\Delta_{\text {edge }}}$, which is valid in the Markovian regime.

\section{APPENDIX B: XXZ INTERACTIONS}

Different strategies have been devised to produce other kinds of spin interactions. One straightforward approach

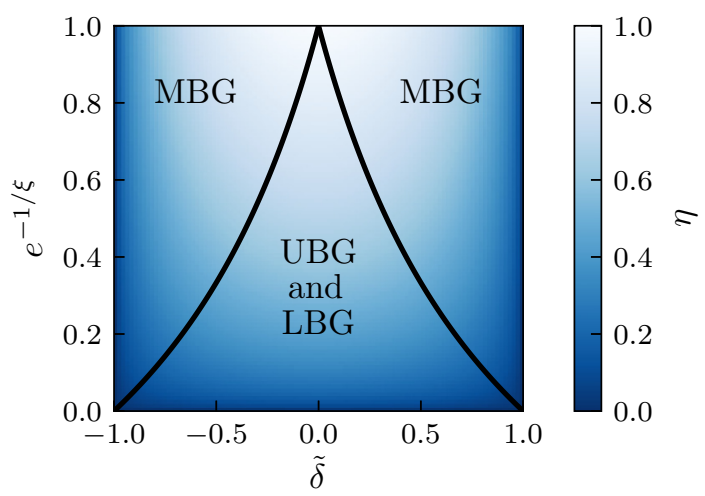

FIG. 14. The allowed values for the parameters $(\xi, \tilde{\delta}, \eta)$ that determine the form of the interactions $J_{n}^{\alpha \beta}$. The black lines delimit the parameter regions that are valid for the upper and lower band gaps (UBG and LBG) and the middle band gap (MBG).

that can be employed whenever fast single-qubit rotations are available is to Trotterize the Hamiltonian [56,118]. Thus, time evolution with a Hamiltonian of the form $H=$ $\tilde{J}\left(a H_{z}+b H_{x y}\right)$, as defined in Eqs. (26), (27), for a short time period $t$ can be approximated by

$$
\begin{aligned}
& e^{i H t} \approx R_{x} e^{i H_{\mathrm{eff}} \frac{a}{4} t} R_{x}^{\dagger} R_{y} e^{i H_{\mathrm{eff}} \frac{(2 b-a)}{4} t} R_{y}^{\dagger} e^{i H_{\mathrm{eff}} \frac{a}{4} t}, \\
& R_{v}=\prod_{n, \alpha} \exp \left[-i \pi S_{n, \alpha}^{v} / 2\right], v \in\{x, y, z\}
\end{aligned}
$$

Note that the term $H_{S}$ in $H_{\text {eff }}$ can be neglected if the evolution is applied to a state with a well-defined number of excitations (it simply produces a phase factor). The smaller the time period $t$ is, the more accurate this approximation will be [119].

Another possibility is to use emitters with a $\Lambda$ level structure, with two metastable states $\left|g_{j}\right\rangle, j=1,2$, an excited state $|e\rangle$, and two lasers driving the $e-g_{1}$ and $e-g_{2}$ transitions, as originally proposed in Ref. [79] (see Fig. 15). In the weak driving regime, when $\Omega_{j} \ll \Delta_{j}$ [we define the detunings $\left.\Delta_{j}=\omega_{e}-\left(\omega_{g_{j}}+\omega_{L j}\right)\right]$, the excited state and the ground-state subspaces can be effectively decoupled. Assuming that only the $e-g_{1}$ transition couples to the waveguide modes, we obtain, to lowest order in perturbation theory, the following effective Hamiltonians for the ground-state subspace:

(a) If $\Delta_{1} \neq \Delta_{2}$,

$$
\begin{aligned}
H_{\mathrm{eff}}= & \sum_{m, n}\left[\left(\frac{\Omega_{1}}{2 \Delta_{1}}\right)^{2}\left(J_{z}\right)_{m n}^{\alpha \beta} \sigma_{11}^{m, \alpha} \sigma_{11}^{n, \beta}\right. \\
& \left.+\left(\frac{\Omega_{2}}{2 \Delta_{2}}\right)^{2}\left(J_{x y}\right)_{m n}^{\alpha \beta} \sigma_{21}^{m, \alpha} \sigma_{12}^{n, \beta}\right],
\end{aligned}
$$




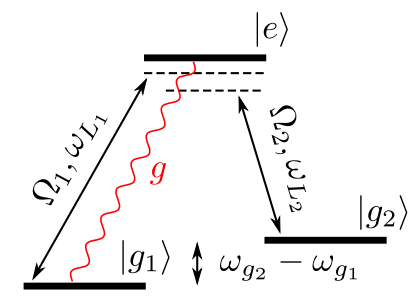

FIG. 15. A schematic depiction of the $\Lambda$ level structure and drivings used in the proposal of Ref. [79]. The $e-g_{1}$ transition couples to the photons in the bath with coupling strength $g$ (indicated by a wiggly red line). The transitions $e-g_{j}$, for $j=1,2$, are driven off resonantly with two independent lasers with amplitudes $\Omega_{j}$ and frequencies $\omega_{L_{j}}$. The two lowest-energy levels can be used to define a spin for each emitter.

where the coupling constants are given by

$$
\begin{gathered}
\left(J_{z}\right)_{m n}^{\alpha \beta}=\operatorname{Re}\left[\Sigma_{m n}^{\alpha \beta}\left(\omega_{e}-\omega_{g_{1}}-\Delta_{1}+i 0^{+}\right)\right], \\
\left(J_{x y}\right)_{m n}^{\alpha \beta}=\operatorname{Re}\left[\Sigma_{m n}^{\alpha \beta}\left(\omega_{e}-\omega_{g_{1}}-\Delta_{2}+i 0^{+}\right)\right],
\end{gathered}
$$

and we use the notation $\sigma_{i j}^{n, \alpha} \equiv\left|g_{i}\right\rangle_{n, \alpha}\left\langle g_{j}\right|$.

(b) If $\Delta_{1}=\Delta_{2}$,

$$
\begin{aligned}
H_{\mathrm{eff}}= & \sum_{m, n} J_{m n}^{\alpha \beta}\left(\frac{\Omega_{1}}{2 \Delta_{1}} \sigma_{11}^{m, \alpha}+\frac{\Omega_{2}}{2 \Delta_{2}} \sigma_{21}^{m, \alpha}\right) \\
& \times\left(\frac{\Omega_{1}}{2 \Delta_{1}} \sigma_{11}^{n, \alpha}+\frac{\Omega_{2}}{2 \Delta_{2}} \sigma_{22}^{n, \alpha}\right) .
\end{aligned}
$$

In this case, the coupling constants are

$$
J_{m n}^{\alpha \beta}=\operatorname{Re}\left[\Sigma_{m n}^{\alpha \beta}\left(\omega_{L_{1}}+i 0^{+}\right)\right] .
$$

Importantly, for these expressions to be valid, we assume the frequency of the $g_{1}-e$ transition to be resonant with some of the band modes. Otherwise, it will produce coherent interactions much stronger than those included in Eqs. (B3) and (B6).

If we define spin operators $S_{n, \alpha}^{+} \equiv \sigma_{21}^{n, \alpha}, S_{n, \alpha}^{-} \equiv \sigma_{12}^{n, \alpha}$, and $S_{n, \alpha}^{z}=\left(\sigma_{11}^{n, \alpha}-\sigma_{22}^{n, \alpha}\right) / 2$, we can readily see that the effective Hamiltonian given in Eq. (B3) is of the XXZ type, albeit the coupling constants in the $x-y$ plane and along the $z$ axis must have a different spatial dependence [cf. Eqs. (B4) and (B5)]. The extent to which a different decay length of the interactions in the $z$ axis versus the $x-y$ plane can affect the many-body phases described in this paper, or whether new phases can arise in this situation, remains an open question. On the other hand, the effective Hamiltonian given in Eq. (B6) contains, besides the interactions of the XXZ model, terms of the type $S_{m, \alpha}^{z} S_{n, \beta}^{-}$, etc., and does not conserve the number of excitations in the emitter subspace. These other spin models remain also largely unexplored.

\section{APPENDIX C: INFINITE-RANGE HAMILTONIAN IN THE UBG AND LBG}

The action of the Hamiltonian in Eq (21) on the states $\left|s_{A}, m_{A} ; s_{B}, m_{B}\right\rangle$, which are eigenstates of $\mathbf{S}_{\alpha}^{2}$ and $S_{\alpha}^{z}$ with eigenvalues $s_{\alpha}\left(s_{\alpha}+1\right)$ and $m_{\alpha}$, respectively $(\alpha=A, B)$, is

$$
\begin{aligned}
& H\left|s_{A}, m_{A} ; s_{B}, m_{B}\right\rangle= \\
& -\tilde{J}\left\{\frac{\sin \theta}{2}\left[s_{A}\left(s_{A}+1\right)-m_{A}^{2}+s_{B}\left(s_{B}+1\right)-m_{B}^{2}\right]+\frac{\cos \theta}{2}\left(m_{A}-m_{B}\right)^{2}+\frac{\mu}{\tilde{J}}\left(m_{A}+m_{B}\right)\right\}\left|s_{A}, m_{A} ; s_{B}, m_{B}\right\rangle \\
& +\tilde{J} \frac{\sin \theta}{2} \sqrt{s_{A}\left(s_{A}+1\right)-m_{A}\left(m_{A}+1\right)} \sqrt{s_{B}\left(s_{B}+1\right)-m_{B}\left(m_{B}-1\right)}\left|s_{A}, m_{A}+1 ; s_{B}, m_{B}-1\right\rangle \\
& +\tilde{J} \frac{\sin \theta}{2} \sqrt{s_{A}\left(s_{A}+1\right)-m_{A}\left(m_{A}-1\right)} \sqrt{s_{B}\left(s_{B}+1\right)-m_{B}\left(m_{B}+1\right)}\left|s_{A}, m_{A}-1 ; s_{B}, m_{B}+1\right\rangle .
\end{aligned}
$$

Thus, the Hamiltonian can be represented by a tridiagonal matrix when acting on the subspace of states with fixed $\left(s_{A}, s_{B}, m=m_{A}+m_{B}\right)$, if a properly sorted basis is chosen. We neglect the permutation quantum numbers, since they do not play any role, but the degeneracy of the ground-state manifold is directly related to them. Numerically, looking for the ground state(s) within each sector with definite total magnetization $m \geq 0$, we find that for $-\pi<\theta<-\pi / 2$, there is a ground state with total angular momenta $s_{A}=s_{B}=\lceil m / 2\rceil(\lceil x\rceil$ denotes the closest integer or half-integer larger than $x$, depending on whether $N / 2$ is even or odd). For $-\pi / 2<\theta<0$, there is a ground state such that $s_{A}=N / 4$ and $s_{B}=|m-N / 4|$, or vice versa. For $0<\theta<\pi$, the ground state has maximum total angular momenta $s_{A}=s_{B}=N / 4$. As can be seen in Fig. 3(b), for $-\pi / 2<\theta<0$, the ground state either has zero total magnetization or is the fully polarized state. So, for $-\pi / 2<$ $\theta<\pi$, the ground state is unique and is fully symmetric 
under permutations of spins within each sublattice. This symmetry entails homogeneous correlations, which do not depend on distance but are different for spins within the same or the opposite sublattice. They can be computed easily once we obtain the ground state, as

$$
\left\langle S_{i, \alpha}^{\nu} S_{j, \beta}^{\nu}\right\rangle= \begin{cases}\frac{4}{N^{2}}\left\langle S_{A}^{\nu} S_{B}^{\nu}\right\rangle, & \text { if } \alpha \neq \beta, \\ \frac{4}{N(N-2)}\left\langle S_{\alpha}^{\nu} S_{\alpha}^{\nu}\right\rangle-\frac{1}{2 N-4}, & \text { if } \alpha=\beta,\end{cases}
$$

where the $S_{\alpha}^{\nu}$ denote the collective spin operators already employed to rewrite the Hamiltonian [Eq. (21)], which have again a simple, at most tridiagonal, matrix representation in the relevant subspace.

For $-\pi<\theta<-\pi / 2$, the ground-state magnetization takes the values $m \in\{0, N / 2\} \quad(m \in\{0,1, N / 2\})$ if $N / 2$ is even (odd) [see Fig. 3(d)]. In these cases, the relevant subspaces have a very small dimension, such that the ground states can be found analytically and with them also the correlations. If $N / 2$ is even, the correlations in the phase with $m=0$ are $\left\langle S_{i, \alpha}^{\nu} S_{j, \alpha}^{\nu}\right\rangle=-1 /(2 N-4),\left\langle S_{i, A}^{\nu} S_{j, B}^{v}\right\rangle=0$. If $N / 2$ is odd, then for $m=0,\left\langle S_{i, \alpha}^{\nu} S_{j, \alpha}^{\nu}\right\rangle=-(1 / 2 N),\left\langle S_{i, A}^{z} S_{j, B}^{z}\right\rangle=-1 / N^{2}$ and $\left\langle S_{i, A}^{x} S_{j, B}^{x}\right\rangle=1 / N^{2}$; whereas for $m=1\left\langle S_{i, \alpha}^{v} S_{j, \alpha}^{v}\right\rangle=$ $-(1 / 2 N),\left\langle S_{i, A}^{z} S_{j, B}^{z}\right\rangle=1 / N^{2}$ and $\left\langle S_{i, A}^{x} S_{j, B}^{x}\right\rangle=0$.

\section{APPENDIX D: NEMATIC AND SPIN-DENSITY-WAVE PHASES}

Our model in the upper band gap with $\tilde{\delta}=0$ and $\theta=$ $-3 \pi / 4$ resembles the zigzag Heisenberg model with ferromagnetic $\mathrm{NN}$ and antiferromagnetic $\mathrm{NNN}$ interactions studied in Ref. [95]. However, our model has longerrange interactions as well and we cannot increase the ratio of NNN to NN interactions without increasing them too. Among all the phases appearing in the zigzag Heisenberg model, the nematic phase and a spin-density wave $\left(\mathrm{SDW}_{2}\right)$ phase also appear in our model. This is demonstrated in Fig. 16, where we show several correlation functions that characterize the different phases:

$$
\begin{aligned}
& C_{2}(r)=\left\langle S_{n-1}^{+} S_{n}^{+} S_{n+r}^{-} S_{n+r+1}^{-}\right\rangle, \\
& C_{3}(r)=\left\langle S_{n-2}^{+} S_{n-1}^{+} S_{n}^{+} S_{n+r}^{-} S_{n+r+1}^{-} S_{n+r+2}^{-}\right\rangle, \\
& C_{\kappa}(r)=\left\langle\kappa_{n} \kappa_{n+r}\right\rangle,
\end{aligned}
$$

where $\kappa_{n}=\left(\mathbf{S}_{n} \times \mathbf{S}_{n+1}\right)^{z}=S_{n}^{x} S_{n+1}^{y}-S_{n}^{y} S_{n+1}^{x}$.

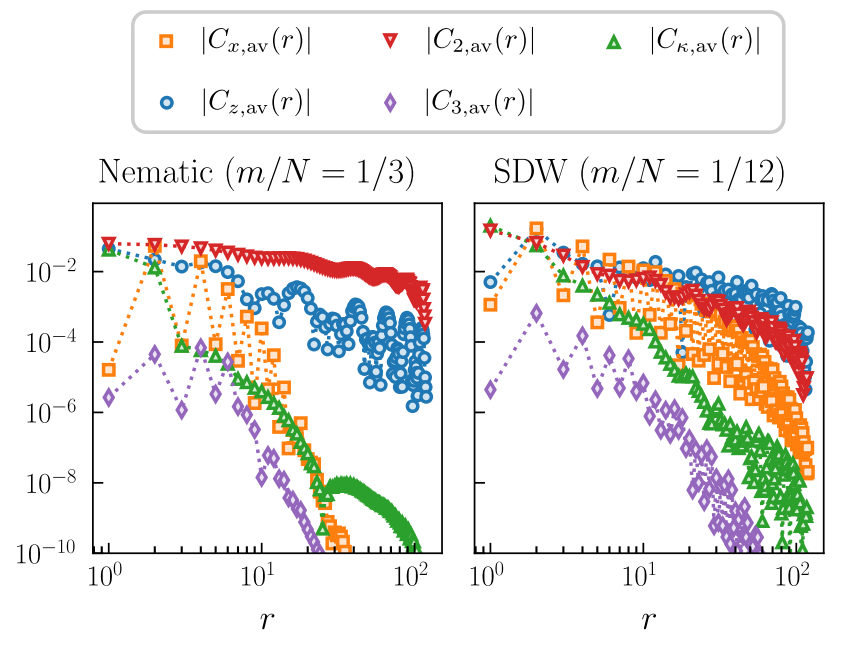

FIG. 16. Different correlation functions for the undimerized model $(\tilde{\delta}=0)$ with $\xi=3$ and $\theta=-3 \pi / 4$, in the upper band gap. The correlations are computed for a chain of length $N=$ 120 , taking the average over the pairs of spins that are closest to the middle of the chain.

\section{APPENDIX E: DIMERIZED XX MODEL}

We would like to solve for the ground state of the following Hamiltonian:

$$
\begin{aligned}
H= & \frac{\tilde{J}}{2} \sum_{j}\left[1+(-1)^{j} \tilde{\delta}\right]\left(S_{j}^{+} S_{j+1}^{-}+S_{j}^{-} S_{j+1}^{+}\right) \\
& -\mu \sum_{j} S_{j}^{z} .
\end{aligned}
$$

As in the main text, we go back and forth between the single-index and two-indices labeling of the different spins. Following Ref. [85], we proceed by applying the Jordan-Wigner transformation:

$$
S_{j}^{-}=c_{j} \prod_{n<j}\left(1-2 c_{n}^{\dagger} c_{n}\right), S_{j}^{z}=c_{j}^{\dagger} c_{j}-1 / 2
$$

The Hamiltonian becomes

$$
\begin{aligned}
H= & \frac{\tilde{J}}{2} \sum_{j}\left[1+(-1)^{j} \tilde{\delta}\right]\left(c_{j}^{\dagger} c_{j+1}+\text { H.c. }\right) \\
& -\mu \sum_{j}\left(c_{j}^{\dagger} c_{j}-\frac{1}{2}\right),
\end{aligned}
$$

which corresponds to a model of free fermions in a 1D lattice. Actually, it is exactly the standard SSH model with a Fermi level set by $\mu$. Calling $a_{j} \equiv c_{2 j} \quad\left(b_{j} \equiv\right.$ $\left.c_{2 j+1}\right)$, we can diagonalize it by Fourier transforming $a_{j}=$ $(N / 2)^{-1 / 2} \sum_{k} e^{i k j} a_{k}\left[b_{j}=(N / 2)^{-1 / 2} \sum_{k} e^{i k j} b_{k}\right]$ (remember that $N$ denotes the number of emitters, which is twice 
the number of unit cells). Its energy spectrum consists of two bands,

$$
H=\sum_{k}\left(\epsilon_{k}^{u} u_{k}^{\dagger} u_{k}+\epsilon_{k}^{l} l_{k}^{\dagger} l_{k}\right)
$$

with dispersion relations and single-particle eigenstates given by

$$
\begin{gathered}
\epsilon_{k}^{u / l}= \pm \frac{\tilde{J}}{2} \sqrt{2\left(1+\delta^{2}\right)+2\left(1-\delta^{2}\right) \cos (k)}-\mu \\
u_{k} / l_{k}=\left( \pm a_{k}+e^{i \phi_{k}} b_{k}\right) / \sqrt{2}
\end{gathered}
$$

with $\phi_{k}=\arg \left((1+\delta)+(1-\delta) e^{-i k}\right)$. The many-body eigenstate with the lowest energy is the one in which all single-particle levels with negative energies are occupied. For $\mu \geq 0$, this implies that excitations over the ground state are created by the following fermionic creation operators:

$$
\eta_{k}^{\dagger}=l_{k}, \xi_{k}^{\dagger}= \begin{cases}u_{k}^{\dagger}, & |k| \leq k_{0}, \\ u_{k}, & |k|>k_{0} .\end{cases}
$$

Here, $k_{0}$ denotes the quasimomentum of the Fermi points, i.e., $k_{0}>0$ such that $\epsilon_{k_{0}}^{u}=0$. This equation has solution only when $\tilde{J}|\delta| \leq \mu \leq \tilde{J}$. Outside of this range, we also define $k_{0}$ as follows for convenience:

$$
k_{0}= \begin{cases}\pi, & 0 \leq \mu \leq \tilde{J}|\tilde{\delta}|, \\ \arccos \left(\frac{2 \mu^{2} / \tilde{J}^{2}-1-\tilde{\delta}^{2}}{1-\tilde{\delta}^{2}}\right), & \tilde{J}|\tilde{\delta}|<\mu<\tilde{J}, \\ 0, & \tilde{J} \leq \mu .\end{cases}
$$

The ground state $|\mathrm{GS}\rangle$ satisfies $\xi_{k}|\mathrm{GS}\rangle=\eta_{k}|\mathrm{GS}\rangle=0$. In the reminder of this appendix, all expectation values are taken with respect to this ground state. The ground-state magnetization is

$$
m=\frac{1}{N} \sum_{j}\left\langle a_{j}^{\dagger} a_{j}+b_{j}^{\dagger} b_{j}-1\right\rangle=\frac{\pi-k_{0}}{2 \pi} .
$$

We can compute correlations between any two spins in the chain as follows:

$$
\begin{aligned}
\left\langle S_{i}^{z} S_{j}^{z}\right\rangle & =\frac{1}{4}\left\langle A_{i} B_{i} A_{j} B_{j}\right\rangle, \\
\left\langle S_{i}^{x} S_{j}^{x}\right\rangle & =\frac{1}{4}\left\langle B_{i}\left(\prod_{n=i+1}^{j-1} A_{n} B_{n}\right) A_{j}\right\rangle,
\end{aligned}
$$

with

$$
A_{i}=c_{i}^{\dagger}+c_{i}, B_{i}=c_{i}^{\dagger}-c_{i} .
$$

This is valid for any 1D spin chain. In our case, we define $A_{i}^{a} / B_{i}^{a} \equiv A_{2 i} / B_{2 i}=a_{i}^{\dagger} \pm a_{i} \quad$ and $\quad A_{i}^{b} / B_{i}^{b} \equiv A_{2 i+1} / B_{2 i+1}$ $=b_{i}^{\dagger} \pm b_{i}$. The contractions of these operators are $\left\langle A_{i}^{\alpha} A_{j}^{\beta}\right\rangle=\delta_{\alpha \beta} \delta_{i j}, \quad\left\langle B_{i}^{\alpha} B_{j}^{\beta}\right\rangle=-\delta_{\alpha \beta} \delta_{i j}, \quad\left\langle B_{i}^{\alpha} A_{j}^{\alpha}\right\rangle=G_{i-j}$, $\left\langle B_{i}^{a} A_{j}^{b}\right\rangle=F_{i-j}$, and $\left\langle B_{i}^{b} A_{j}^{a}\right\rangle=F_{j-i}$, with

$$
\begin{aligned}
G_{r} & \equiv \frac{1}{\pi} \int_{k_{0}}^{\pi} d k \cos (k r)= \begin{cases}1-k_{0} / \pi, & r=0, \\
-\frac{\sin \left(k_{0} r\right)}{\pi r}, & r \neq 0,\end{cases} \\
F_{r} & \equiv-\frac{1}{\pi} \int_{0}^{k_{0}} d k \cos \left(k r+\phi_{k}\right) .
\end{aligned}
$$

Using Wick's theorem, we can now compute

$$
\begin{aligned}
\left\langle S_{i}^{z} S_{j}^{z}\right\rangle & =\frac{\left\langle B_{i} A_{i}\right\rangle\left\langle B_{j} A_{j}\right\rangle-\left\langle B_{i} A_{j}\right\rangle\left\langle B_{j} A_{j}\right\rangle}{4}, \\
\left\langle S_{i}^{x} S_{j}^{x}\right\rangle & =\frac{\operatorname{det} M}{4}
\end{aligned}
$$

with

$$
M=\left(\begin{array}{cccc}
\left\langle B_{i} A_{i+1}\right\rangle & \left\langle B_{i} A_{i+2}\right\rangle & \cdots & \left\langle B_{i} A_{j}\right\rangle \\
\left\langle B_{i+1} A_{i+1}\right\rangle & \left\langle B_{i+1} A_{i+2}\right\rangle & \cdots & \left\langle B_{i+1} A_{j}\right\rangle \\
\vdots & \vdots & \ddots & \\
\left\langle B_{j-1} A_{i+1}\right\rangle & \left\langle B_{j-1} A_{i+2}\right\rangle & & \left\langle B_{j-1} A_{j}\right\rangle
\end{array}\right)
$$

The dimer order parameter, Eq. (23) particularized for $p=$ 2 , can be computed as

$$
\begin{aligned}
O_{2} & =\frac{\left\langle\mathbf{S}_{n, B} \mathbf{S}_{n+1, A}\right\rangle-\left\langle\mathbf{S}_{n, A} \mathbf{S}_{n, B}\right\rangle}{2} \\
& =\frac{F_{0}^{2}-F_{1}^{2}+2\left(F_{1}-F_{0}\right)}{8} .
\end{aligned}
$$

\section{APPENDIX F: NON-ABELIAN MANY-BODY BERRY PHASES}

As discussed in Refs. [68,70,120], the many-body Berry phases obtained for certain specific twists of the Hamiltonian can be interpreted as topological invariants characterizing different SPT orders. For this, one has to choose an appropriate modulation of the Hamiltonian, such that the associated Berry phases are quantized due to the symmetries of the phase we want to characterize.

Let us apply this theory to the $\mathrm{VBS}_{q}$ phases discussed in the main text. As we show, a suitable twist to characterize these phases consist in the modulation of the complex phase of the exchange coupling constant between two spins in different sublattices: $e^{-i \phi} S_{m, A}^{+} S_{n, B}^{-}+$H.c.. If the ground state is unique and it is symmetric about the middle of the link connecting these two spins, then the Berry 
phase,

$$
\gamma=-\int_{0}^{2 \pi} d \phi\left\langle\operatorname{GS}(\phi)\left|i \partial_{\phi}\right| \mathrm{GS}(\phi)\right\rangle
$$

obtained for the ground state $|\mathrm{GS}(\phi)\rangle$ of the modulated Hamiltonian $H(\phi)$, must be quantized. To prove this, let us also consider the complementary path in which $\phi$ goes from $2 \pi$ to 0 and the associated Berry phase $\gamma^{\prime}$. Since the concatenation of both paths yields the trivial path, we have that $\gamma+\gamma^{\prime}=0(\bmod 2 \pi)$. On the other hand, for chains with PBCs, $H(-\phi)=U_{I} H(\phi) U_{I}^{\dagger}$, where $U_{I}$ is the (nonlocal) unitary associated to the space-inversion transformation about the middle of the link connecting the two spins. If, for all values of $\phi$, the system remains gaped and the ground state is unique, the ground states along each path are related to each other through a gauge transformation, $|\mathrm{GS}(-\phi)\rangle=U_{I}|\mathrm{GS}(\phi)\rangle=e^{i \Omega(\phi)}|\mathrm{GS}(\phi)\rangle$, which, in turn, implies that $\gamma=\gamma^{\prime}(\bmod 2 \pi)$. Combined, these two conditions restrict the possible values of $\gamma$ to 0 or $\pi$ $(\bmod 2 \pi)$.

A similar derivation can be obtained for non-Abelian Berry phases in the case of a degenerate ground state [121]. This is the one we use to characterize the ground state in the $\mathrm{VBS}_{q}$ phases, for $q>2$. Numerically, it can be computed by discretizing the path, choosing a set of points $\left\{\phi_{n}\right\}_{n=1, \ldots, N}$ such that $0 \leq \phi_{n}<\phi_{n+1}<2 \pi$, and computing the matrix of overlaps $\Phi_{n}$, the components of which are $\left(\Phi_{n}\right)_{\mu \nu}=\left\langle\mathrm{GS}_{\nu}\left(\phi_{n}\right) \mid \mathrm{GS}_{\mu}\left(\phi_{n+1}\right)\right\rangle$, where $\left\{\left|\mathrm{GS}_{\mu}\left(\phi_{n}\right)\right\rangle\right\}_{\mu=1, \ldots, q / 2}$ denotes the ground-state multiplet at each point along the path [we identify $\left|\mathrm{GS}_{\mu}\left(\phi_{N+1}\right)\right\rangle=$ $\left.\left|\mathrm{GS}_{\mu}\left(\phi_{1}\right)\right\rangle\right]$. Then, the non-Abelian Berry phase can be computed as $\gamma=-\sum_{n} \arg \left(\operatorname{det} \Phi_{n}\right)$. Even though a single many-body ground state may be periodic with a period larger than the period of the Hamiltonian, when we compute these non-Abelian Berry phases, we recover the original periodicity of the Hamiltonian, so that there are just two inequivalent many-body Berry phases between nearest-neighbor spins in chains with PBCs: $\gamma_{\text {inter }}$ and $\gamma_{\text {intra }}$. Furthermore, since changing the sign of the dimerization parameter is equivalent to a redefinition of the unit cell, it is clear that upon changing $\tilde{\delta} \rightarrow-\tilde{\delta}$, the two Berry phases interchange: $\gamma_{\text {inter }} \leftrightarrow \gamma_{\text {intra }}$.

[1] A. Auerbach, Interacting Electrons and Quantum Magnetism, Graduate Texts in Contemporary Physics( Springer-Verlag, New York, 1994).

[2] P. A. Lee, N. Nagaosa, and X.-G. Wen, Doping a Mott insulator: Physics of high-temperature superconductivity, Rev. Mod. Phys. 78, 17 (2006).

[3] R. M. Clegg, The history of FRET: From conception through the labors of birth, Reviews in Fluorescence (2006).

[4] S. Sachdev, Quantum Phase Transitions (2011).
[5] A. Lucas, Ising formulations of many NP problems, Front. Phys. 2, 1 (2014).

[6] P. Henelius and A. W. Sandvik, Sign problem in Monte Carlo simulations of frustrated quantum spin systems, Phys. Rev. B 62, 1102 (2000).

[7] J. I. Cirac and P. Zoller, Goals and opportunities in quantum simulation, Nat. Phys. 8, 264 (2012).

[8] D. Porras and J. I. Cirac, Effective Quantum Spin Systems with Trapped Ions, Phys. Rev. Lett. 92, 207901 (2004).

[9] P. Nevado and D. Porras, Hidden frustrated interactions and quantum annealing in trapped-ion spin-phonon chains, Phys. Rev. A 93, 13625 (2016).

[10] A. Friedenauer, H. Schmitz, J. T. Glueckert, D. Porras, and T. Schaetz, Simulating a quantum magnet with trapped ions, Nat. Phys. 4, 757 (2008).

[11] H. Kim, S. M. Thon, P. M. Petroff, and D. Bouwmeester, Independent tuning of quantum dots in a photonic crystal cavity, Appl. Phys. Lett. 95, 243107 (2009).

[12] K. Kim, M.-S. Chang, S. Korenblit, R. Islam, E. E. Edwards, J. K. Freericks, G.-D. Lin, L.-M. Duan, and C. Monroe, Quantum simulation of frustrated Ising spins with trapped ions, Nature 465, 590 (2010).

[13] E. E. Edwards, S. Korenblit, K. Kim, R. Islam, M.S. Chang, J. K. Freericks, G.-D. Lin, L.-M. Duan, and C. Monroe, Quantum simulation and phase diagram of the transverse-field Ising model with three atomic spins, Phys. Rev. B 82, 060412 (2010).

[14] R. Islam, E. E. Edwards, K. Kim, S. Korenblit, C. Noh, H. Carmichael, G.-D. Lin, L.-M. Duan, C.-C. Joseph Wang, J. K. Freericks, and C. Monroe, Onset of a quantum phase transition with a trapped ion quantum simulator, Nat. Commun. 2, 377 (2011).

[15] G.-D. Lin, C. Monroe, and L.-M. Duan, Sharp Phase Transitions in a Small Frustrated Network of Trapped Ion Spins, Phys. Rev. Lett. 106, 230402 (2011).

[16] S. Sachdev, K. Sengupta, and S. M. Girvin, Mott insulators in strong electric fields, Phys. Rev. B 66, 075128 (2002).

[17] C.-M. Simon, T. Belhadj, B. Chatel, T. Amand, P. Renucci, A. Lemaitre, O. Krebs, P. A. Dalgarno, R. J. Warburton, X. Marie, and B. Urbaszek, Robust Quantum Dot Exciton Generation via Adiabatic Passage with Frequency-Swept Optical Pulses, Phys. Rev. Lett. 106, 166801 (2011).

[18] R. Ma, M. E. Tai, P. M. Preiss, W. S. Bakr, J. Simon, and M. Greiner, Photon-Assisted Tunneling in a Biased Strongly Correlated Bose Gas, Phys. Rev. Lett. 107, 95301 (2011).

[19] F. Meinert, M. J. Mark, E. Kirilov, K. Lauber, P. Weinmann, A. J. Daley, and H.-C. Nägerl, Quantum Quench in an Atomic One-Dimensional Ising Chain, Phys. Rev. Lett. 111, 053003 (2013).

[20] F. Meinert, M. J. Mark, E. Kirilov, K. Lauber, P. Weinmann, M. Gröbner, A. J. Daley, and H. C. Nägerl, Observation of many-body dynamics in long-range tunneling after a quantum quench, Science 344, 1259 (2014).

[21] L.-M. Duan, E. Demler, and M. D. Lukin, Controlling Spin Exchange Interactions of Ultracold Atoms in Optical Lattices, Phys. Rev. Lett. 91, 90402 (2003).

[22] J. J. García-Ripoll and J. I. Cirac, Spin dynamics for bosons in an optical lattice, New. J. Phys. 5, 76 (2003). 
[23] A. B. Kuklov and B. V. Svistunov, Counterflow Superfluidity of Two-Species Ultracold Atoms in a Commensurate Optical Lattice, Phys. Rev. Lett. 90, 100401 (2003).

[24] S. Trotzky, P. Cheinet, S. Fölling, M. Feld, U. Schnorrberger, A. M. Rey, A. Polkovnikov, E. A. Demler, M. D. Lukin, and I. Bloch, Time-resolved observation and control of superexchange interactions with ultracold atoms in optical lattices, Science 319, 295 (2008).

[25] S. Nascimbène, Y.-A. Chen, M. Atala, M. Aidelsburger, S. Trotzky, B. Paredes, and I. Bloch, Experimental Realization of Plaquette Resonating Valence-Bond States with Ultracold Atoms in Optical Superlattices, Phys. Rev. Lett. 108, 205301 (2012).

[26] T. Fukuhara, A. Kantian, M. Endres, M. Cheneau, P. Schauß, S. Hild, D. Bellem, U. Schollwöck, T. Giamarchi, C. Gross, I. Bloch, and S. Kuhr, Quantum dynamics of a mobile spin impurity, Nat. Phys. 9, 235 (2013).

[27] T. Fukuhara, P. Schauß, M. Endres, S. Hild, M. Cheneau, I. Bloch, and C. Gross, Microscopic observation of magnon bound states and their dynamics, Nature 502, 76 (2013).

[28] S. Hild, T. Fukuhara, P. Schauß, J. Zeiher, M. Knap, E. Demler, I. Bloch, and C. Gross, Far-from-Equilibrium Spin Transport in Heisenberg Quantum Magnets, Phys. Rev. Lett. 113, 147205 (2014).

[29] H. Weimer, M. Müller, I. Lesanovsky, P. Zoller, and H. P. Büchler, A Rydberg quantum simulator, Nat. Phys. 6, 382 (2010).

[30] I. Lesanovsky, Many-Body Spin Interactions and the Ground State of a Dense Rydberg Lattice Gas, Phys. Rev. Lett. 106, 025301 (2011).

[31] H. Labuhn, D. Barredo, S. Ravets, S. de Léséleuc, T. Macrì, T. Lahaye, and A. Browaeys, Tunable twodimensional arrays of single Rydberg atoms for realizing quantum Ising models, Nature 534, 667 (2016).

[32] H. Bernien, S. Schwartz, A. Keesling, H. Levine, A. Omran, H. Pichler, S. Choi, A. S. Zibrov, M. Endres, M. Greiner, others, Probing many-body dynamics on a 51-atom quantum simulator, Nature 551, 579 (2017).

[33] S. De Léséleuc, V. Lienhard, P. Scholl, D. Barredo, S. Weber, N. Lang, H. P. Büchler, T. Lahaye, and A. Browaeys, Observation of a symmetry-protected topological phase of interacting bosons with Rydberg atoms, Science 365, 775 (2019).

[34] A. Browaeys and T. Lahaye, Many-body physics with individually controlled Rydberg atoms, Nat. Phys. 16, 132 (2020).

[35] E. Vetsch, D. Reitz, G. Sagué, R. Schmidt, S. T. Dawkins, and A. Rauschenbeutel, Optical Interface Created by Laser-Cooled Atoms Trapped in the Evanescent Field Surrounding an Optical Nanofiber, Phys. Rev. Lett. 104, 203603 (2010).

[36] A. Goban, C.-L. Hung, S.-P. Yu, J. D. Hood, J. A. Muniz, J. H. Lee, M. J. Martin, A. C. McClung, K. S. Choi, D. E. Chang, O. Painter, and H. J. Kimblemblrm, Atom-light interactions in photonic crystals, Nat. Commun. 5, 3808 (2014).

[37] J. D. Thompson, T. G. Tiecke, N. P. de Leon, J. Feist, A. V. Akimov, M. Gullans, A. S. Zibrov, V. Vuletic, and M. D. Lukin, Coupling a single trapped atom to a nanoscale optical cavity, Science 340, 1202 (2013).
[38] J.-B. Béguin, E. M. Bookjans, S. L. Christensen, H. L. S \ orensen, J. H. Müller, E. S. Polzik, and J. Appel, Generation and Detection of a Sub-Poissonian Atom Number Distribution in a One-Dimensional Optical Lattice, Phys. Rev. Lett. 113, 263603 (2014).

[39] A. Goban, C.-L. Hung, J. D. Hood, S.-P. Yu, J. A. Muniz, O. Painter, and H. J. Kimble, Superradiance for Atoms Trapped along a Photonic Crystal Waveguide, Phys. Rev. Lett. 115, 63601 (2015).

[40] J. D. Hood, A. Goban, A. Asenjo-Garcia, M. Lu, S.-P. Yu, D. E. Chang, and H. J. Kimble, Atom-atom interactions around the band edge of a photonic crystal waveguide, Proc. Natl. Acad. Sci. 113, 10507 (2016).

[41] P. Solano, B. D. Patterson, S. L. Rolston, L. A. Orozco, F. K. Fatemi, J. P. Clemens, P. R. Rice, P. Barberis-Blostein, and H. J. Carmichael, in Frontiers in Optics 2016 (Optical Society of America, 2016), p. LW5I.4.

[42] M. E. Kim, T.-H. Chang, B. M. Fields, C.-A. Chen, and C.-L. Hung, Trapping single atoms on a nanophotonic circuit with configurable tweezer lattices, Nat. Commun. 10, 1647 (2019).

[43] X. Luan, J.-B. Béguin, A. P. Burgers, Z. Qin, S.-P. Yu, and H. J. Kimble, The integration of photonic crystal waveguides with atom arrays in optical tweezers, Adv. Quantum Technol. 3, 2000008 (2020).

[44] P. Samutpraphoot, T. Dordević, P. L. Ocola, H. Bernien, C. Senko, V. Vuletić, and M. D. Lukin, Strong Coupling of two Individually Controlled Atoms via a Nanophotonic Cavity, Phys. Rev. Lett. 124, 063602 (2020).

[45] A. Laucht, S. Pütz, T. Günthner, N. Hauke, R. Saive, S. Frédérick, M. Bichler, M.-C. Amann, A. W. Holleitner, M. Kaniber, and J. J. Finley, A Waveguide-Coupled On-Chip Single-Photon Source, Phys. Rev. X 2, 11014 (2012).

[46] P. Lodahl, S. Mahmoodian, and S. Stobbe, Interfacing single photons and single quantum dots with photonic nanostructures, Rev. Mod. Phys. 87, 347 (2015).

[47] R. E. Evans, M. K. Bhaskar, D. D. Sukachev, C. T. Nguyen, A. Sipahigil, M. J. Burek, B. Machielse, G. H. Zhang, A. S. Zibrov, E. Bielejec, H. Park, M. Lončar, and M. D. Lukin, Photon-mediated interactions between quantum emitters in a diamond nanocavity, Science 362, 662 (2018).

[48] A. F. van Loo, A. Fedorov, K. Lalumière, B. C. Sanders, A. Blais, and A. Wallraff, Photon-mediated interactions between distant artificial atoms, Science 342, 1494 (2013).

[49] Y. Liu and A. A. Houck, Quantum electrodynamics near a photonic bandgap, Nat. Phys. 13, 48 (2017).

[50] M. Mirhosseini, E. Kim, V. S. Ferreira, M. Kalaee, A. Sipahigil, A. J. Keller, and O. Painter, Superconducting metamaterials for waveguide quantum electrodynamics, Nat. Commun. 9, 3706 (2018).

[51] M. Mirhosseini, E. Kim, X. Zhang, A. Sipahigil, P. B. Dieterle, A. J. Keller, A. Asenjo-Garcia, D. E. Chang, and O. Painter, Cavity quantum electrodynamics with atom-like mirrors, Nature 569, 692 (2019).

[52] E. Kim, X. Zhang, V. S. Ferreira, J. Banker, J. K. Iverson, A. Sipahigil, M. Bello, A. González-Tudela, M. Mirhosseini, and O. Painter, Quantum Electrodynamics in a Topological Waveguide, Phys. Rev. X 11, 011015 (2021). 
[53] L. Krinner, M. Stewart, A. Pazmino, J. Kwon, and D. Schneble, Spontaneous emission of matter waves from a tunable open quantum system, Nature 559, 589 (2018).

[54] J. S. Douglas, H. Habibian, C.-L. Hung, A. V. Gorshkov, H. J. Kimble, and D. E. Chang, Quantum many-body models with cold atoms coupled to photonic crystals, Nat. Photonics 9, 326 (2015).

[55] A. González-Tudela, C.-L. Hung, D. E. Chang, J. I. Cirac, and H. J. Kimble, Subwavelength vacuum lattices and atom-atom interactions in two-dimensional photonic crystals, Nat. Photonics 9, 320 (2015).

[56] C.-L. Hung, A. González-Tudela, J. I. Cirac, and H. J. Kimble, Quantum spin dynamics with pairwise-tunable, long-range interactions, Proc. Natl. Acad. Sci. 113, E4946 (2016).

[57] D. E. Chang, J. S. Douglas, A. González-Tudela, C.-L. Hung, and H. J. Kimble, Colloquium: Quantum matter built from nanoscopic lattices of atoms and photons, Rev. Mod. Phys. 90, 031002 (2018).

[58] M. Bello, G. Platero, J. I. Cirac, and A. González-Tudela, Unconventional quantum optics in topological waveguide QED, Sci. Adv. 5, eaaw0297 (2019).

[59] L. Leonforte, A. Carollo, and F. Ciccarello, VacancyLike Dressed States in Topological Waveguide QED, Phys. Rev. Lett. 126, 063601 (2021).

[60] A. Saxena, Y. Chen, Z. Fang, and A. Majumdar, Photonic topological baths for quantum simulation, arXiv:2106.14325 [quant-ph] (2021).

[61] V. P. Bykov, Spontaneous emission from a medium with a band spectrum, Soviet J. Quantum Electron. 4, 861 (1975).

[62] S. John and J. Wang, Quantum Electrodynamics near a Photonic Band gap: Photon Bound States and Dressed Atoms, Phys. Rev. Lett. 64, 2418 (1990).

[63] G. Kurizki, Two-atom resonant radiative coupling in photonic band structures, Phys. Rev. A 42, 2915 (1990).

[64] M. T. Manzoni, L. Mathey, and D. E. Chang, Designing exotic many-body states of atomic spin and motion in photonic crystals, Nat. Commun. 8, 14696 (2017).

[65] D. Z. Li, M. T. Manzoni, and D. E. Chang, Random singlet phase of cold atoms coupled to a photonic crystal waveguide, 2020.

[66] U. Schollwöck, The density-matrix renormalization group in the age of matrix product states, Ann. Phys. (N.Y.) 326, 96 (2011).

[67] J. Hauschild and F. Pollmann, Efficient numerical simulations with tensor networks: Tensor network PYTHON (TeNPy), SciPost Phys. Lecture Notes 5, 005 (2018).

[68] Y. Hatsugai, Quantized Berry phases as a local order parameter of a quantum liquid, J. Phys. Soc. Jpn 75, 123601 (2006).

[69] T. Hirano, H. Katsura, and Y. Hatsugai, Topological classification of gapped spin chains: Quantized Berry phase as a local order parameter, Phys. Rev. B 77, 094431 (2008).

[70] T. Kariyado, T. Morimoto, and Y. Hatsugai, $Z_{N}$ Berry Phases in Symmetry Protected Topological Phases, Phys. Rev. Lett. 120, 247202 (2018).

[71] P. Nevado, S. Fernández-Lorenzo, and D. Porras, Topological Edge States in Periodically Driven Trapped-Ion Chains, Phys. Rev. Lett. 119, 210401 (2017).
[72] Z.-X. Gong, M. F. Maghrebi, A. Hu, M. L. Wall, M. FossFeig, and A. V. Gorshkov, Topological phases with longrange interactions, Phys. Rev. B 93, 41102 (2016).

[73] K. Patrick, T. Neupert, and J. K. Pachos, Topological Quantum Liquids with Long-Range Couplings, Phys. Rev. Lett. 118, 267002 (2017).

[74] A. Bermudez, L. Tagliacozzo, G. Sierra, and P. Richerme, Long-range Heisenberg models in quasiperiodically driven crystals of trapped ions, Phys. Rev. B 95, 024431 (2017).

[75] C. Cohen-Tannoudji, J. Dupont-Roc, and G. Grynberg, Atom-Photon Interactions (1998).

[76] P. Hauke, F. M. Cucchietti, A. Müller-Hermes, M.-C. Bañuls, J. I. Cirac, and M. Lewenstein, Complete Devil's staircase and crystal-superfluid transitions in a dipolar XXZ spin chain: A trapped ion quantum simulation, New J. Phys. 12, 113037 (2010).

[77] I. de Vega, D. Porras, and J. I. Cirac, Matter-Wave Emission in Optical Lattices: Single Particle and Collective Effects, Phys. Rev. Lett. 101, 260404 (2008).

[78] C. Navarrete-Benlloch, I. de Vega, D. Porras, and J. I. Cirac, Simulating quantum-optical phenomena with cold atoms in optical lattices, New J. Phys. 13, 23024 (2011).

[79] A. González-Tudela, V. Paulisch, D. Chang, H. Kimble, and J. Cirac, Deterministic Generation of Arbitrary Photonic States Assisted by Dissipation, Phys. Rev. Lett. 115, 163603 (2015).

[80] H. Ritsch, P. Domokos, F. Brennecke, and T. Esslinger, Cold atoms in cavity-generated dynamical optical potentials, Rev. Mod. Phys. 85, 553 (2013).

[81] E. J. Davis, G. Bentsen, L. Homeier, T. Li, and M. H. Schleier-Smith, Photon-Mediated Spin-Exchange Dynamics of Spin-1 Atoms, Phys. Rev. Lett. 122, 010405 (2019).

[82] U. Schollwöck, J. Richter, D. J. J. Farnell, and R. F. Bishop, (Editors)Quantum Magnetism (2004).

[83] N. Ishimura and H. Shiba, Dynamical correlation functions of one-dimensional anisotropic Heisenberg model with Spin 1/2. I: Ising-like antiferromagnets, Prog. Theor. Phys. 63, 743 (1980).

[84] J. Des Cloizeaux and M. Gaudin, Anisotropic linear magnetic chain, J. Math. Phys. 7, 1384 (1966).

[85] E. Lieb, T. Schultz, and D. Mattis, Two soluble models of an antiferromagnetic chain, Ann. Phys. (N.Y.) 16, 407 (1961).

[86] T. Giamarchi, Quantum Physics in One Dimension (Oxford University Press, Oxford, 2003).

[87] F. T. Arecchi, E. Courtens, R. Gilmore, and H. Thomas, Atomic coherent states in quantum optics, Phys. Rev. A 6 , 2211 (1972).

[88] P. Bak and R. Bruinsma, One-Dimensional Ising Model and the Complete Devil's Staircase, Phys. Rev. Lett. 49, 249 (1982).

[89] F. D. M. Haldane, Spontaneous dimerization in the $S=$ $\frac{1}{2}$ Heisenberg antiferromagnetic chain with competing interactions, Phys. Rev. B 25, 4925 (1982).

[90] T. Hikihara, M. Kaburagi, and H. Kawamura, Groundstate phase diagrams of frustrated spin- $S X X Z$ chains: Chiral ordered phases, Phys. Rev. B 63, 174430 (2001). 
[91] K. Okunishi and T. Tonegawa, Magnetic phase diagram of the $S=1 / 2$ antiferromagnetic zigzag spin chain in the strongly frustrated region: Cusp and plateau, J. Phys. Soc. Jpn 72, 479 (2003).

[92] I. P. McCulloch, R. Kube, M. Kurz, A. Kleine, U. Schollwöck, and A. K. Kolezhuk, Vector chiral order in frustrated spin chains, Phys. Rev. B 77, 094404 (2008).

[93] A. W. Sandvik, in AIP Conference Proceedings (2010).

[94] For closed boundary conditions, the two possible dimerization patterns are degenerate and the observed $\delta B^{v}$ may be zero. Still, this phase is characterized by the longrange order of the bond-bond correlation functions $D(r)=$ $\left\langle B_{n}^{v} B_{n+r}^{v}\right\rangle$.

[95] T. Hikihara, L. Kecke, T. Momoi, and A. Furusaki, Vector chiral and multipolar orders in the spin-1/2 frustrated ferromagnetic chain in magnetic field, Phys. Rev. B 78, 144404 (2008).

[96] J. Asbóth, L. Oroszlány, and A. Pályi, A Short Course on Topological Insulators: Band Structure and Edge States in One and Two Dimensions, Lecture Notes in Physics (Springer International Publishing, 2016).

[97] R. Verresen, R. Moessner, and F. Pollmann, Onedimensional symmetry protected topological phases and their transitions, Phys. Rev. B 96, 165124 (2017).

[98] F. Pollmann, A. M. Turner, E. Berg, and M. Oshikawa, Entanglement spectrum of a topological phase in one dimension, Phys. Rev. B 81, 064439 (2010).

[99] T. L. Hughes, E. Prodan, and B. A. Bernevig, Inversionsymmetric topological insulators, Phys. Rev. B 83, 245132 (2011).

[100] R. Verresen, R. Thorngren, N. G. Jones, and F. Pollmann, Gapless Topological Phases and Symmetry-Enriched Quantum Criticality, Phys. Rev. X 11, 041059 (2021).

[101] J. Fraxanet, D. González-Cuadra, T. Pfau, M. Lewenstein, T. Langen, and L. Barbiero, Topological quantum critical points in the extended Bose-Hubbard model, arXiv:2106.15457 [cond-mat.quant-gas] (2021).

[102] A. P. Burgers, L. S. Peng, J. A. Muniz, A. C. McClung, M. J. Martin, and H. J. Kimble, Clocked atom delivery to a photonic crystal waveguide, Proc. Natl. Acad. Sci. U.S.A. 116, 456 (2019).

[103] X. Luan, J. Béguin, A. P. Burgers, Z. Qin, S. Yu, and H. J. Kimble, The integration of photonic crystal waveguides with atom arrays in optical tweezers, Adv. Quantum Technol. 3, 2000008 (2020).

[104] J. B. Beguin, J. Laurat, X. Luan, A. P. Burgers, Z. Qin, and H. J. Kimble, Reduced volume and reflection for bright optical tweezers with radial Laguerre-Gauss beams, Proc. Natl. Acad. Sci. U.S.A. 117, 26109 (2020).

[105] N. Fayard, A. Bouscal, J. Berroir, A. Urvoy, T. Ray, S. Mahapatra, M. Kemiche, J.-A. Levenson, J.-J. Greffet, K. Bencheikh, J. Laurat, and C. Sauvan, Asymmetric comb waveguide for strong interactions between atoms and light, arXiv:2201.02507 (2022).

[106] T. Dordevic, P. Samutpraphoot, P. L. Ocola, H. Bernien, B. Grinkemeyer, I. Dimitrova, V. Vuletić, and M. D. Lukin,
Entanglement transport and a nanophotonic interface for atoms in optical tweezers, Science 373, 1511 (2021).

[107] S. D. Mishra, R. Trivedi, A. H. Safavi-Naeini, and J. Vučković, Control design for inhomogeneous broadening compensation in single-photon transducers, arXiv:2012. 01718 [quant-ph] (2021).

[108] D. Levonian, R. Riedinger, B. Machielse, E. Knall, M. Bhaskar, C. Knaut, R. Bekenstein, H. Park, M. Loncar, and M. Lukin, Optical Entanglement of Distinguishable Quantum Emitters, arXiv:2108.10928 (2021).

[109] E. N. Knall, C. M. Knaut, R. Bekenstein, D. R. Assumpcao, P. L. Stroganov, W. Gong, Y. Q. Huan, P.-J. Stas, B. Machielse, M. Chalupnik, D. Levonian, A. Suleymanzade, R. Riedinger, H. Park, M. Lončar, M. K. Bhaskar, and M. D. Lukin, Efficient Source of Shaped Single Photons Based on an Integrated Diamond Nanophotonic System, (2022).

[110] M. H. Appel, A. Tiranov, A. Javadi, M. C. Löbl, Y. Wang, S. Scholz, A. D. Wieck, A. Ludwig, R. J. Warburton, and P. Lodahl, Coherent Spin-Photon Interface with Waveguide Induced Cycling Transitions, Phys. Rev. Lett. 126, 013602 (2021).

[111] P. Delplace, D. Ullmo, and G. Montambaux, Zak phase and the existence of edge states in graphene, Phys. Rev. B 84, 195452 (2011).

[112] T. Albash and D. A. Lidar, Adiabatic quantum computation, Rev. Mod. Phys. 90, 015002 (2018).

[113] A. T. Rezakhani, W.-J. Kuo, A. Hamma, D. A. Lidar, and P. Zanardi, Quantum Adiabatic Brachistochrone, Phys. Rev. Lett. 103, 080502 (2009).

[114] R. Kimmel and J. A. Sethian, Computing geodesic paths on manifolds, Proc. Natl. Acad. Sci. 95, 8431 (1998).

[115] G. Peyré, M. Péchaud, R. Keriven, and L. D. Cohen, Geodesic methods in computer vision and graphics, Foundations Trends Computer Graphics Vision 5, 197 (2010).

[116] C. Vega, M. Bello, D. Porras, and A. González-Tudela, Qubit-photon bound states in topological waveguides with long-range hoppings, arXiv:2105.12470 (2021).

[117] H.-P. Breuer and F. Petruccione, The Theory of Open Quantum Systems (Oxford University Press, Oxford, 2007), p. 656.

[118] U. L. Heras, A. Mezzacapo, L. Lamata, S. Filipp, A. Wallraff, and E. Solano, Digital Quantum Simulation of Spin Systems in Superconducting Circuits, Phys. Rev. Lett. 112, 200501 (2014).

[119] A. M. Childs, Y. Su, M. C. Tran, N. Wiebe, and S. Zhu, Theory of Trotter Error with Commutator Scaling, Phys. Rev. X 11, 011020 (2021).

[120] T. Kariyado and Y. Hatsugai, Fractionally quantized Berry phase, adiabatic continuation, and edge states, Phys. Rev. B 90, 085132 (2014).

[121] F. Wilczek and A. Zee, Appearance of Gauge Structure in Simple Dynamical Systems, Phys. Rev. Lett. 52, 2111 (1984). 